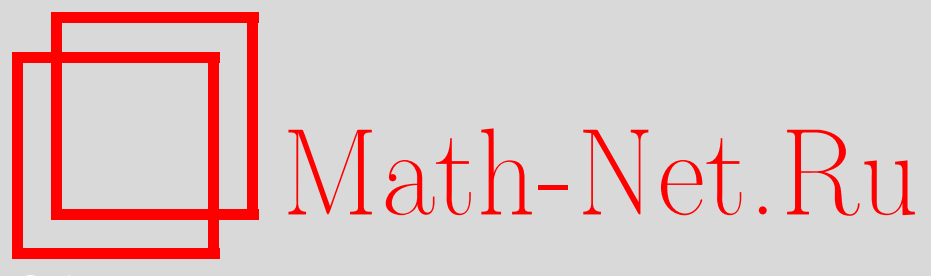

В. В. Жиков, Об оценках типа Нэша-Аронсона для уравнения диффузии с несимметрической матрицей и их приложении к усреднению, Матем. сб., 2006, том 197, номер 12, 65-94

DOI: https://doi.org/10.4213/sm1472

Использование Общероссийского математического портала Math-Net.Ru подразумевает, что вы прочитали и согласны с пользовательским соглашением http: //www . mathnet.ru/rus/agreement

Параметры загрузки:

IP: 35.174 .16 .151

26 апреля 2023 г., 12:56:35

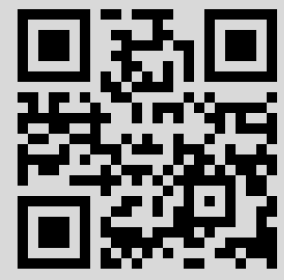




\author{
В. В. Жиков
}

\title{
Об оценках типа Нэша-Аронсона для уравнения диффузии с несимметрической матрицей и их приложении к усреднению
}

Рассматривается параболическое уравнение с несимметрическим эллиптическим оператором второго порядка. Доказываются оценки типа Нэша-Аронсона в том случае, когда кососимметрическая часть матрицы теплопроводности не является ограниченной. Указанные оценки применяются при доказательстве центральной предельной теоремы для диффузии в несжимаемом случайном потоке.

Библиография: 19 названий.

\section{$\S$ 1. Введение}

1.1. Рассмотрим задачу Коши

$$
\frac{\partial u}{\partial t}=\operatorname{div} A(x) \nabla u,\left.\quad u\right|_{t=0}=f \in L^{2}\left(\mathbb{R}^{d}\right),
$$

где измеримая не обязательно симметрическая матрица $A(x)$ удовлетворяет условию эллиптичности:

$$
\nu \xi^{2} \leqslant A(x) \xi \cdot \xi \leqslant \nu^{-1} \xi^{2}, \quad \nu>0 .
$$

Условие (1.2) не налагает никаких ограничений на кососимметрическую часть $B=\left(A-A^{T}\right) / 2$ матрицы $A$.

Известно [1]-[3], что если $B \in L^{\infty}\left(\mathbb{R}^{d}\right)$, то задаче (1.1) соответствует действующая в $L^{2}\left(\mathbb{R}^{d}\right)$ сильно непрерывная полугруппа операторов $e^{-t \mathscr{A}}, \mathscr{A}=$ $-\operatorname{div} A \nabla$. При этом $e^{-t \mathscr{A}}$ есть интегральный оператор и соответствующее ядро $K(x, y, t)$ (фундаментальное решение) удовлетворяет оценке Нэша-Аронсона

$$
0 \leqslant K(x, y, t) \leqslant \frac{c_{0}}{t^{d / 2}} e^{-(x-y)^{2} /\left(c_{0} t\right)}
$$

в которой константа $c_{0}>0$ зависит только от $d, \nu$ и $\|B\|_{L^{\infty}}$.

Наряду с оценкой (1.3) для фундаментального решения представляет интерес оценка вида

$$
|u(x, t)| \leqslant \frac{c_{0}}{t^{d / 2}} e^{c_{0} t \lambda^{2}-\lambda|x|} \int_{\mathbb{R}^{d}} e^{\lambda|s|}|f(s)| d s,
$$

содержащая произвольный вещественный параметр $\lambda$. Если в этой оценке перейти к начальному значению $f(x)=\delta(x)$ (дельта-функция в точке $x=0$ ), а в показателе взять минимум по $\lambda$, то придем к оценке (1.3) при $y=0$. Оценки с параметром предпочтительней, когда имеются те или иные затруднения с построением фундаментального решения.

(C) В. В. ЖКиков, 2006 
Мы рассматриваем случай, когда матрица $B$ не является локально ограниченной, а лишь суммируемой с некоторой степенью. В этих условиях сталкиваемся с обескураживающим явлением - решение задачи Коши (1.1) определено не единственным образом. Позже обсудим проблему единственности и определим так называемую аппроксимационную полугруппу, а сейчас сформулируем ключевой результат, в котором сама проблема единственности не затрагивается.

Речь идет о некоторых оценках для решения задачи Коши и фундаментального решения, полученных в предположении ограниченности матрицы $B$. Важно, однако, что константы в этих оценках зависят не от $\|B\|_{L^{\infty}\left(\mathbb{R}^{d}\right)}$, а лишь от величин $p>d$ и $l$,

$$
l=\sup _{r \geqslant 1} \frac{1}{r^{d}} \int_{|x| \leqslant r}|B|^{p} d x<\infty .
$$

Введем функцию

$$
g(\lambda)=\lambda^{2}+|\lambda|^{\alpha}, \quad \lambda \in \mathbb{R}^{1}, \quad \alpha=\frac{2 p}{p-d},
$$

и сопряженную к ней функцию $g^{*}(k)=\sup _{\lambda}\{\lambda k-g(\lambda)\}$.

ТЕОРема 1.1. В предположении ограниченности матрицы В для решения задачи Коши (1.1) и фундаментального решения справедливы оценки

$$
\begin{gathered}
\sup _{x \in \mathbb{R}^{d}}\left|\left(x^{2}+1\right)^{\lambda} u(x, t)\right| \leqslant \frac{c_{0}}{t^{d / 2}} e^{c_{0} t g(\lambda)} \int_{\mathbb{R}^{d}}\left(s^{2}+1\right)^{\lambda}|f(s)| d s, \\
0 \leqslant K(x, 0, t) \leqslant \frac{c_{0}}{t^{d / 2}} e^{-c_{0} t g^{*}\left(\ln \left(x^{2}+1\right) /\left(c_{0} t\right)\right)} \equiv \Phi(x, t) .
\end{gathered}
$$

Здесъ $\lambda \in \mathbb{R}^{1}$ произвольно, а постоянная с с зависит только от размерности пространства, константы эллиптичности $\nu$ ивеличин $p>d u l$ из (1.4). Например, можно взять

$$
c_{0}=c(d) \nu^{-(p+d) /(p-d)} l^{2 /(p-d)} .
$$

В случае, когда матрица $B$ удовлетворяет лишь условию (1.4) (т.е. не является ограниченной), существование решения, удовлетворяющего оценке (1.5), будет получено путем аппроксимации.

По определению фундаментального решения $\lim _{t \rightarrow 0} K=\delta(x)$ в смысле слабой сходимости мер, где $\delta(x)$ - дельта-функция. Оценка (1.6) обеспечивает равномерный характер этой сходимости, а именно справедливость соотношения

$$
\int_{|x| \geqslant r} K(x, 0, t) d x \leqslant c_{r}(t), \quad \lim _{t \rightarrow 0} c_{r}(t)=0 \quad \forall r>0,
$$

в которых функция $c_{r}(t)$ определяется только величинами $d, \nu, p, l$. Действительно, достаточно заметить, что $g(\lambda) \leqslant 2|\lambda|^{\alpha}+1, g^{*}(\eta) \geqslant \gamma|\eta|^{\alpha^{\prime}}-1$, где $\gamma>0$, $\alpha^{\prime}=\alpha /(\alpha-1)>1$, и поэтому

$$
\Phi(x, t) \leqslant \frac{c_{0}}{t^{d / 2}} \exp \left[-\gamma \frac{\ln ^{\alpha^{\prime}}\left(x^{2}+1\right)}{\left(c_{0} t\right)^{1 /(\alpha-1)}}\right] .
$$


Отсюда легко следует, что

$$
\lim _{t \rightarrow 0} \int_{|x| \geqslant r} \Phi(x, t) d x=0 \quad \forall r>0 .
$$

В теории усреднения рассматривается задача Коши (1.1), в которой вместо матрицы $A(x)$ берется матрица $A\left(\varepsilon^{-1} x\right)$. Поскольку для матрицы $B\left(\varepsilon^{-1} x\right)$ $(0<\varepsilon \leqslant 1)$ основное условие (1.4) выполнено с той же константой $l$, оценки $(1.5),(1.6)$ будут равномерными по $\varepsilon$. Это обстоятельство играет ключевую роль при доказательстве так называемой центральной предельной теоремы, cм. $\S 6$.

Возьмем в оценке (1.5) $\lambda=0$. Тогда получим, что

$$
\sup _{x \in \mathbb{R}^{d}}|u(x, t)| \leqslant \frac{c_{0}}{t^{d / 2}} \int_{\mathbb{R}^{d}}|f(s)| d s .
$$

Стоит отметить, что константу $c_{0}$ в этом случае можно выбрать зависящей только от размерности пространства и постоянной эллиптичности, т.е. никак не зависящей от матрицы $B$.

Оценка (1.6), очевидно, обеспечивает степенное убывание ядра $K$ при фиксированном $t>0$ и $|x| \rightarrow \infty$. Чтобы добиться экспоненциального убывания, нужны более жесткие, чем (1.4), требования на матрицу $B$. Пусть для некоторого $p>d$

$$
\sup _{\square}\|B\|_{L^{p}(\square)}=l<\infty
$$

где $\square=[0,1]^{d}+y$ - единичный куб, а супремум берется по всем таким кубам.

Очевидно, что условие (1.8) (в отличие от (1.4)) сохраняется не только при гомотетии, но и при сдвиге.

ТЕОРема 1.2. В предположении ограниченности матричы В справедливы оценки

$$
\begin{gathered}
\sup _{x \in \mathbb{R}^{d}}\left|e^{\lambda|x|} u(x, t)\right| \leqslant \frac{c_{0}}{t^{d / 2}} e^{c_{0} t g(\lambda)} \int_{\mathbb{R}^{d}} e^{\lambda|s|}|f(s)| d s \\
0 \leqslant K(x, y, t) \leqslant \frac{c_{0}}{t^{d / 2}} e^{-c_{0} t g^{*}\left(|x-y| /\left(t c_{0}\right)\right)} .
\end{gathered}
$$

Здесъ $\lambda \in \mathbb{R}^{1}$ произвольно, а постоянная с 0 зависит только от размерности пространства, константы эллиптичности $\nu$ и величин $l$ и $p$ из (1.8).

Отметим, что при $p \rightarrow \infty$ условие (1.8) переходит в условие $B \in L^{\infty}\left(\mathbb{R}^{d}\right)$, а оценка (1.9) - в классическую оценку Нэша-Аронсона (1.3).

Приведенные оценки можно записать в единой форме

$$
|u(x, t)| \leqslant \frac{c_{0}}{t^{d / 2}} e^{c_{0} t g(\lambda)-\lambda \psi_{0}(x)} \int_{\mathbb{R}^{d}} e^{\lambda \psi_{0}(s)}|f(s)| d s,
$$

где в качестве $\psi_{0}$ следует взять одну из функций $\ln \left(x^{2}+1\right),|x|$. Если в этой оценке перейти к начальному значению $f(x)=\delta(x)$, а в показателе взять инфимум по $\lambda \in \mathbb{R}^{1}$, то получим

$$
0 \leqslant K(x, 0, t) \leqslant \frac{c_{0}}{t^{d / 2}} e^{-c_{0} t g^{*}\left(\psi_{0}(x) /\left(c_{0} t\right)\right)} .
$$


По ходу доказательства оценки (1.10) мы устанавливаем также "весовую" энергетическую оценку

$$
\begin{aligned}
\int_{\mathbb{R}^{d}} e^{2 \lambda \psi_{0}(x)}|u(x, t)|^{2} d x+\frac{\nu}{2} \int_{0}^{t} \int_{\mathbb{R}^{d}} e^{2 \lambda \psi_{0}(x)}|\nabla u(x, s)|^{2} d x d s \\
\leqslant\left(1+\lambda^{2} t\right) e^{c_{0} t g(\lambda)} \int_{\mathbb{R}^{d}} e^{2 \lambda \psi_{0}(s)}|f(s)|^{2} d s \quad \forall \lambda \in \mathbb{R}^{1} .
\end{aligned}
$$

Сделаем одно замечание относительно указанных выше оценок. Все они опираются на условие (1.4) (или на более сильное условие (1.8)). Ослабим условие (1.4), взяв в нем $p \leqslant d$. Тогда никаких оценок такого рода доказать не удается, а из некоторых результатов по усреднению можно заключить, что свойство (1.7) равномерного дельтаобразного поведения в этом случае не имеет места, другими словами, подходящих оценок просто нет. Строгое обоснование будет дано в $\S 3$, п. 3.2. Однако условие (1.4) можно ослабить в ином отношении, а именно с сохранением оценки (1.5) заменить на

$$
\int_{1}^{\infty} \frac{1}{r^{p+1}} \int_{|x| \leqslant r}|B|^{p} d x d r=l<\infty,
$$

где по-прежнему $p>d$. Например, если $|B(x)| \leqslant C\left(|x|^{k}+1\right)$, где $0<k<1$, то это условие выполнено с любым $p>d /(1-k)$. Если же матрица $B$ линейна по $x, B(x) \not \equiv$ const, то условие (1.12) не выполняется, а оценка (1.5) теряет силу. Последнее будет пояснено ниже.

1.2. Обсудим теперь проблему единственности. Начнем с эллиптического уравнения

$$
-\operatorname{div} A \nabla u+u=f \in L^{2}\left(\mathbb{R}^{d}\right) .
$$

Скажем, что функция $u \in H^{1}\left(\mathbb{R}^{d}\right)$ есть решение этого уравнения, если

$$
\int_{\mathbb{R}^{d}}[a \nabla u \cdot \nabla \varphi+B \nabla u \cdot \nabla \varphi+u \varphi] d x=\int_{\mathbb{R}^{d}} f \varphi d x \quad \forall \varphi \in C_{0}^{\infty}\left(\mathbb{R}^{d}\right),
$$

где $a$ - симметрическая часть матрицы $A, a=\left(A+A^{T}\right) / 2$.

Данное определение имеет смысл, если $B \in L_{\text {loc }}^{2}\left(\mathbb{R}^{d}\right)$. Это минимальное требование обеспечивает и существование решения. В самом деле, рассмотрим аппроксимацию

$$
B_{n} \in L^{\infty}\left(\mathbb{R}^{d}\right), \quad B_{n} \text { кососимметрична, } \quad B_{n} \rightarrow B \text { в } L_{\mathrm{loc}}^{2}\left(\mathbb{R}^{d}\right),
$$

при сохранении симметрической части $a$. Пусть $u_{n}$ - решение соответствующего уравнения,

$$
\begin{gathered}
u_{n} \in H^{1}\left(\mathbb{R}^{d}\right), \\
\int_{\mathbb{R}^{d}}\left[a \nabla u_{n} \cdot \nabla \varphi+B_{n} \nabla u_{n} \cdot \nabla \varphi+u_{n} \varphi\right] d x=\int_{\mathbb{R}^{d}} f \varphi d x \quad \forall \varphi \in C_{0}^{\infty}\left(\mathbb{R}^{d}\right) .
\end{gathered}
$$

В исходном тождестве (1.14) вообще нельзя было взять в качестве пробной функции само решение $u$. Теперь это возможно и, полагая в $(1.15) \varphi=u_{n}$, в силу кососимметричности $B_{n}$ получим энергетическое равенство

$$
\int_{\mathbb{R}^{d}}\left(a \nabla u_{n} \cdot \nabla u_{n}+u_{n}^{2}\right) d x=\int_{\mathbb{R}^{d}} f u_{n} d x
$$


и энергетическую оценку

$$
\left\|u_{n}\right\|_{H^{1}\left(\mathbb{R}^{d}\right)} \leqslant \nu^{-1}\|f\|_{L^{2}\left(\mathbb{R}^{d}\right)} .
$$

Видим, что последовательность $u_{n}$ ограничена в $H^{1}$, и можно считать, что она слабо сходится $u_{n} \rightarrow u$ в $H^{1}$. Тогда в тождестве (1.15) легко перейти к пределу и получить тождество (1.14).

Таким образом, процедура аппроксимации всякий раз приводит к решению исходного уравнения (1.13). Такие решения называются аппроксимационными. Проблема единственности рассмотрена в [4], в частности там указано эллиптическое уравнение, которое помимо аппроксимационного решения имеет и другое решение, которое не может быть получено никакой аппроксимацией.

Стоит отметить, что для аппроксимационного решения выполнено "энергетическое неравенство"

$$
\int_{\mathbb{R}^{d}}\left(a \nabla u \cdot \nabla u+u^{2}\right) d x \leqslant \int_{\mathbb{R}^{d}} f u d x .
$$

Это непосредственно следует из энергетического равенства для $u_{n}$ и полунепрерывности стоящего слева квадратичного функционала относительно слабой сходимости $u_{n} \rightarrow u$ в $H^{1}\left(\mathbb{R}^{d}\right)$. Справедлива также энергетическая оценка

$$
\|u\|_{H^{1}\left(\mathbb{R}^{d}\right)} \leqslant \nu^{-1}\|f\|_{L^{2}\left(\mathbb{R}^{d}\right)} .
$$

Хотя существование аппроксимационного решения уравнения (1.13) установлено, главный вопрос, будет ли последовательность эллиптических операторов $\mathscr{A}_{n}=-\operatorname{div}\left(a+B_{n}\right) \nabla$ компактной в смысле сильной резольвентной сходимости, остался пока не выясненным. Сейчас мы дадим ответ на этот вопрос сразу в терминах сходимости соответствующих полугрупп.

Напомним, что $u(x, t)$ есть решение задачи Коши (1.1), если

$$
u, \nabla u \in L^{2}\left(\mathbb{R}^{d} \times[0, T]\right) \quad \forall T>0
$$

и выполнено интегральное тождество

$$
\int_{0}^{\infty} \int_{\mathbb{R}^{d}} u \frac{\partial \varphi}{\partial t} d x d t+\int_{\mathbb{R}^{d}} f \varphi d x=\int_{0}^{\infty} \int_{\mathbb{R}^{d}} A \nabla u \cdot \nabla \varphi d x d t \quad \forall \varphi \in C_{0}^{\infty}\left(\mathbb{R}^{d+1}\right) .
$$

При условии $B \in L_{\text {loc }}^{2}\left(\mathbb{R}^{d}\right)$ существование аппроксимационного решения доказывается столь же просто, как и в случае эллиптического уравнения (1.13). Однако заранее не вполне очевидно, что аппроксимационные решения можно задать с помощью полугрупп.

Теорема 1.3. Пусть матрица В удовлетворяет условию

$$
\sup _{r \geqslant 1} \frac{1}{r^{d}} \int_{|x| \leqslant r}|B|^{d} d x=l<\infty
$$

a аппроксимачии $B_{n}$ таковь, что

$$
B_{n} \in L^{\infty}\left(\mathbb{R}^{d}\right), \quad B_{n} \rightarrow B \quad \text { в } \quad L_{\mathrm{loc}}^{d}\left(\mathbb{R}^{d}\right), \quad \sup _{n} \sup _{r \geqslant 1} \frac{1}{r^{d}} \int_{|x| \leqslant r}\left|B_{n}\right|^{d} d x<\infty .
$$


Тогда последовательность полугрупп $e^{-t \mathscr{A}_{n}}$ компактна в смысле сильной $L^{2}$ сходимости при любом $t \geqslant 0$. Иначе говоря, с точностью до выбора подпоследовательности

$$
e^{-t \mathscr{A}_{n}} f \rightarrow U(t) f \quad \text { в } \quad L^{2}\left(\mathbb{R}^{d}\right) \quad \forall f \in L^{2}\left(\mathbb{R}^{d}\right),
$$

где $U(t)$ - сильно непрерьвная полугруппа в $L^{2}\left(\mathbb{R}^{d}\right)$.

Видим, что в условиях теоремы 1.3 любое (подходящим образом определенное) аппроксимационное решение $u(\cdot, t)$ задачи Коши имеет структуру $u(\cdot, t)=$ $U(t) f$, где $U(t)$ - некоторая "аппроксимационная" полугруппа. Позже (см. §5) укажем дополнительные условия, при которых аппроксимационная полугруппа единственна.

Разумеется, при выполнении условия (1.17) последовательность операторов $\mathscr{A}_{n}$ будет компактной в смысле сильной резольвентной сходимости. Для предельного оператора $\mathscr{A}$ (он служит производящим оператором "аппроксимационной" полугруппы $U(t)$, см. (1.19)) уравнение $\mathscr{A} u+u=f$ имеет единственное решение, и это решение будет аппроксимационным решением эллиптического уравнения (1.12).

Обсудим оценки (1.10), (1.11) для аппроксимационных решений. Предполагаем, что аппроксимации $B_{n}$ удовлетворяют условию (1.4) равномерно по $n$ и $B_{n} \rightarrow B$ в $L_{\text {loc }}^{p}\left(\mathbb{R}^{d}\right)$. Возьмем начальное значение $f \in C_{0}^{\infty}\left(\mathbb{R}^{d}\right)$, и пусть $u_{n}(\cdot, t)=$ $e^{-t \mathscr{A}_{n}} f, u(\cdot, t)=U(t) f$. Тогда непосредственно из соотношения (1.19) ясно, что оценка (1.10) сохраняется для $u(x, t)$. Относительно оценки (1.11) необходимо еще учесть слабую сходимость градиентов $\nabla u_{n} \rightarrow \nabla u$ в $L^{2}\left(\mathbb{R}^{d} \times[0, T]\right)$. После того как оценки $(1.10),(1.11)$ установлены для $f \in C_{0}^{\infty}\left(\mathbb{R}^{d}\right)$, по замыканию получаем сильно непрерывную полугруппу $U(t)$ в $L^{2}\left(\mathbb{R}^{d}, e^{2 \lambda \psi_{0}} d x\right)$, а при $t>0$ - непрерывный оператор $U(t)$ из $L^{1}\left(\mathbb{R}^{d}, e^{2 \lambda \psi_{0}} d x\right)$ в $L^{\infty}\left(\mathbb{R}^{d}, e^{2 \lambda \psi_{0}} d x\right)$. Для $u(\cdot, t)=U(t) f$ оценки $(1.10),(1.11)$ будут, очевидно, выполнены.

Вопрос о гёльдеровости и даже просто непрерывности аппроксимационного решения при $t>0$ остался открытым. По-видимому, здесь не обойтись без дополнительных условий на матрицу $B$, например принадлежности $B$ пространству функций с ограниченным средним колебанием (BMO) или сверхэкспоненциальной суммируемости (это значит, что $e^{s|B|} \in L_{\text {loc }}^{1}\left(\mathbb{R}^{d}\right)$ при любом $s>0$ ). Оба эти условия обсуждались в [4] в связи с проблемой единственности.

1.3. Поясним особую роль кососимметрической матрицы $B$ с точки зрения диффузии. Пусть матрица $B$ является достаточно гладкой, а именно имеет дивергенцию $b=\operatorname{div} B \in L_{\mathrm{loc}}^{1}\left(\mathbb{R}^{d}\right)$, т.е.

$$
b_{j} \in L_{\mathrm{loc}}^{1}\left(\mathbb{R}^{d}\right), \quad \int_{\mathbb{R}^{d}} b_{j} h_{j} d x=-\int_{\mathbb{R}^{d}} B_{i j} \frac{\partial h_{j}}{\partial x_{i}} d x \quad \forall h_{j} \in C_{0}^{\infty}\left(\mathbb{R}^{d}\right) .
$$

Вектор $b$ автоматически соленоидален, $\operatorname{div} b=0$, что непосредственно следует из кососимметричности матрицы $B$. Если дополнительно

$$
b \in L_{\mathrm{loc}}^{2 d /(d+2)}\left(\mathbb{R}^{d}\right) \quad \text { при } \quad d \geqslant 3, \quad b \in L_{\text {loc }}^{1+\sigma}\left(\mathbb{R}^{2}\right), \sigma>0, \quad \text { при } \quad d=2,
$$

то справедливо тождество

$$
\int_{\mathbb{R}^{d}} B \nabla u \cdot \nabla \varphi d x=\int_{\mathbb{R}^{d}} b u \cdot \nabla \varphi d x \quad \forall \varphi \in C_{0}^{\infty}\left(\mathbb{R}^{d}\right) .
$$


Действительно, сначала проверяем его для $u \in C_{0}^{\infty}\left(\mathbb{R}^{d}\right)$, а затем переходим к пределу, пользуясь тем, что $B \nabla u \in L_{\text {loc }}^{1}$ и $b u \in L_{\text {loc }}^{1}$ в силу (1.21) и теоремы вложения Соболева. Тождество (1.22) позволяет переписать уравнение (1.1) в виде

$$
\frac{\partial u}{\partial t}=\operatorname{div}(a \nabla u+b u) .
$$

Обратно, если дано уравнение с соленоидальным вектором $b$, удовлетворяющим условию (1.21), то, представив последний в виде дивергенции от кососимметрической матрицы $B \in L_{\mathrm{loc}}^{2}\left(\mathbb{R}^{d}\right)$, получим уравнение с матрицей $B$. Например, в случае $d=2$ имеем

$$
B=\left(\begin{array}{cc}
0 & \beta(x) \\
-\beta(x) & 0
\end{array}\right), \quad b=\operatorname{div} B=\left\{-\frac{\partial \beta}{\partial x_{2}}, \frac{\partial \beta}{\partial x_{1}}\right\} .
$$

Отметим, что замена уравнения с вектором $b$ на уравнение с матрицей $B$ автоматически желаемых результатов не дает - требуется обеспечить еще соответствующие свойства матрицы $B$. Начнем с "хорошего" случая. Пусть вектор $b$ периодичен,

$$
\int_{\square} b d x=0, \quad b \in L^{q}(\square) \text { для некоторого } q>\frac{d}{2},
$$

где $\square$ - ячейка периодичности. Тогда справедливо представление $b=\operatorname{div} B$, в котором периодическая матрица принадлежит $L^{p}(\square), p>d$. Видим, что условие (1.8) выполнено.

Дело обстоит иначе, если вектор $b$ (вектор сноса) постоянен, $b \neq 0$, поскольку соответствующая матрица $B$ линейна и условие (1.4) не выполняется. В таком случае целесообразно от решения $u(x, t)$ перейти к функции $w(x, t)=u(x-b t, t)$, которая удовлетворяет дивергентному уравнению без сноса, но с матрицей $a(x-b t)$, зависящей, как видно, и от аргумента $t$. В связи с этим вообще имеет смысл рассмотреть оценки типа Нэша-Аронсона в случае, когда в (1.1) матрица $A$ зависит от $x$ и $t$. В настоящей работе нет возможности подробно остановиться на этом вопросе. Как бы то ни было, если матрица $а$ постоянна, для $w$ справедлива оценка (1.5), откуда легко заключить, что для исходной функции $u(x, t)$ она не выполняется.

Оценкам типа Нэша-Аронсона посвящена обширная литература. Изучались главным образом параболические уравнения с симметрической эллиптической частью, например уравнение теплопроводности в области евклидова пространства с условием Неймана на границе, а также уравнение теплопроводности на многообразии, см. [5]-[7]. В работе [8] имеется некоторый обзор применяемых подходов, а также значительная библиография. Книга [9] содержит много материала по оценкам Нэша-Аронсона и библиографию до 1989 г.

Уравнение (1.23) с соленоидальным вектором $b$, очевидно, описывает диффузию в несжимаемом потоке. Интерес к уравнению с неограниченной кососимметрической матрицей вызван тем, что именно такому уравнению отвечает диффузия в турбулентном потоке. Обсуждение связи с турбулентностью и дальнейшие по этому вопросу ссылки имеются в [10]-[14]. В случае неограниченной матрицы $B$ каких-либо оценок типа Нэша-Аронсона ранее доказано не было, хотя многократно отмечалось, что их отсутствие является основным препятствием к доказательству центральной предельной теоремы в наиболее желательной форме, см. §6, теорема 6.3. 


\section{§ 2. Об ограниченности снизу некоторых эллиптических операторов}

По заданной на $\mathbb{R}^{d}$ достаточно гладкой функции $\psi(x)$ строим оператор

$$
\mathscr{A}_{\psi}=e^{\psi} \mathscr{A} e^{-\psi}, \quad \text { где } \quad \mathscr{A}=-\operatorname{div} A \nabla, \quad A=a+B, \quad B \in L^{\infty}\left(\mathbb{R}^{d}\right) .
$$

Очевидно, что

$$
-\mathscr{A}_{\psi} v=\operatorname{div} A \nabla v-\nabla \psi \cdot A \nabla v-\operatorname{div}(v A \nabla \psi)+\nabla \psi \cdot A \nabla \psi v .
$$

Здесь и далее запись вида $\nabla \psi v$ надо понимать как $v \nabla \psi$, а не $\nabla(\psi v)$. Для билинейной формы соответствующего оператора в $L^{2}\left(\mathbb{R}^{d}\right)$ имеем равенство

$$
\begin{aligned}
\left(\mathscr{A}_{\psi} v, w\right)_{L^{2}}= & \int_{\mathbb{R}^{d}} A \nabla v \cdot \nabla w d x+\int_{\mathbb{R}^{d}} \nabla \psi \cdot A \nabla v w d x \\
& \quad-\int_{\mathbb{R}^{d}} v A \nabla \psi \cdot \nabla w d x-\int_{\mathbb{R}^{d}} a \nabla \psi \cdot \nabla \psi v w d x .
\end{aligned}
$$

Функция $\psi$ будет специально выбираться в зависимости от того, какое из двух условий (1.4) или (1.8) рассматривается.

2.1. Начнем с основного условия (1.4) и положим

$$
\psi(x)=\lambda \psi_{0}(x), \quad \psi_{0}(x)=\ln \left(x^{2}+1\right), \quad \lambda \in \mathbb{R}^{1} .
$$

В данном случае $e^{\psi}=\left(x^{2}+1\right)^{\lambda}, \nabla \psi_{0}=2 x /\left(x^{2}+1\right)$ и требуется оценить снизу квадратичную форму

$$
\left(\mathscr{A}_{\psi} v, v\right)_{L^{2}}=\int_{\mathbb{R}^{d}} a \nabla v \cdot \nabla v d x-2 \lambda \int_{\mathbb{R}^{d}} B \nabla \psi_{0} \cdot \nabla v v d x-\lambda^{2} \int_{\mathbb{R}^{d}} a \nabla \psi_{0} \cdot \nabla \psi_{0} v^{2} d x .
$$

Ниже используется обозначение

$$
\|v\|_{q}=\left(\int_{\mathbb{R}^{d}}|v|^{q} d x\right)^{1 / q}, \quad 1 \leqslant q \leqslant \infty .
$$

Лемма 2.1. Справедлива оценка

$$
I \equiv \lambda \int_{\mathbb{R}^{d}} B \nabla \psi_{0} \cdot \nabla v v d x \leqslant \frac{\nu}{8}\|\nabla v\|_{2}^{2}+c_{0}|\lambda|^{\alpha}\|v\|_{2}^{2}, \quad \alpha=\frac{2 p}{p-d},
$$

в которой постоянная $c_{0}$ зависит толъко от $d, \nu$ и величин $p>d$ u $l$ из (1.4).

ДоказАтельство. Убедимся, что

$$
\left\|B \nabla \psi_{0}\right\|_{p} \leqslant c_{1}, \quad c_{1}=c_{1}(p, l) .
$$

Действительно, положим

$$
\eta(r)=\left(\frac{2 r}{r^{2}+1}\right)^{p}, \quad G(r)=\int_{|x| \leqslant r}|B|^{p} d x .
$$

Так как $\left|\nabla \psi_{0}\right| \leqslant 2|x| /\left(x^{2}+1\right)$, то в сферических координатах имеем

$$
\int_{|x| \leqslant R}\left|\nabla \psi_{0}\right|^{p}|B|^{p} d x \leqslant \int_{0}^{R} \eta(r) \frac{d}{d r} G(r) d r=\eta(R) G(R)-\int_{0}^{R} G(r) \eta^{\prime}(r) d r .
$$


Первое слагаемое справа стремится к нулю при $R \rightarrow \infty$ в силу условия (1.4), поэтому

$$
\int_{\mathbb{R}^{d}}\left|\nabla \psi_{0}\right|^{p}|B|^{p} d x=-\int_{0}^{\infty} G(r) \eta^{\prime}(r) d r
$$

и неравенство (2.4) следует из (1.4).

Далее используем известное мультипликативное неравенство

$$
\|u\|_{q} \leqslant C\|\nabla u\|_{2}^{\theta}\|u\|_{2}^{1-\theta}, \quad \theta=\left(\frac{1}{2}-\frac{1}{q}\right) d, \quad 2 \leqslant q<\frac{2 d}{d-2},
$$

где $C=C(d, q)$, см. [15; гл. II, §2]. Определим показатель $q$ из соотношения $1 / p+1 / 2+1 / q=1$. В этом случае $\theta=d / p$ и по неравенству Гёльдера

$$
I \leqslant|\lambda|\left\|B \nabla \psi_{0}\right\|_{p}\|\nabla v\|_{2}\|v\|_{q} \leqslant c_{1}|\lambda|\|\nabla v\|_{2}\|v\|_{q} \leqslant c_{2}|\lambda|\|\nabla v\|_{2}^{1+\theta}\|v\|_{2}^{1-\theta} .
$$

Так как по неравенству Юнга $a b \leqslant a^{2 /(1+\theta)}+b^{2 /(1-\theta)}$, где $a, b \geqslant 0$, то

$$
I \leqslant c_{2}|\lambda|\left(t^{2 /(1+\theta)}\|\nabla v\|_{2}^{2}+t^{-2 /(1-\theta)}\|v\|_{2}^{2}\right) \quad \forall t>0 .
$$

Выбирая $t$ из соотношения $c_{2}|\lambda| t^{2 /(1+\theta)}=\nu / 8$, после несложных вычислений получим оценку (2.3). Лемма 2.1 доказана.

Теперь из (2.2) следует искомая оценка снизу

$$
\left(\mathscr{A}_{\psi} v, v\right)_{L^{2}} \geqslant \frac{\nu}{2}\|\nabla v\|_{2}^{2}-\frac{c_{1}}{2} g(\lambda)\|v\|_{2}^{2}
$$

в которой постоянная $c_{1}$ зависит только от $d, \nu$ и величин $p$ и $l$ из (1.4).

2.2. Нам требуется обобщить оценку (2.6), а именно оценить снизу форму $J=\left(\mathscr{A}_{\psi} v, w\right)_{L^{2}}, \quad w=s|v|^{2 s-2} v, s \geqslant 1$. Для упрощения записи считаем, что $v \geqslant 0$. Тогда $w=s v^{2 s-1}$ и из (2.1) имеем

$J=s \int_{\mathbb{R}^{d}}\left[A \nabla v \cdot \nabla v^{2 s-1}+\lambda \nabla \psi_{0} \cdot A \nabla v v^{2 s-1}-\lambda v A \nabla \psi_{0} \cdot \nabla v^{2 s-1}-\lambda^{2} a \nabla \psi_{0} \cdot \nabla \psi_{0} v^{2 s}\right] d x$.

Учитывая, что

$$
\begin{gathered}
A \nabla v \cdot \nabla\left(s v^{2 s-1}\right)=\frac{2 s-1}{s} A \nabla v^{s} \cdot \nabla v^{s}=\frac{2 s-1}{s} a \nabla v^{s} \cdot \nabla v^{s}, \\
s \nabla \psi_{0} \cdot A \nabla v v^{2 s-1}=v^{s} \nabla \psi_{0} \cdot A \nabla v^{s}, \\
s v A \nabla \psi_{0} \cdot \nabla v^{2 s-1}=(2 s-1) v^{s} A \nabla \psi_{0} \cdot \nabla v^{s},
\end{gathered}
$$

получим

$$
\begin{aligned}
J=\int_{\mathbb{R}^{d}} & {\left[\frac{2 s-1}{s} a \nabla v^{s} \cdot \nabla v^{s}-\lambda 2(s-1) a \nabla v^{s} \cdot \nabla \psi_{0} v^{s}\right.} \\
& \left.+2 s \lambda v^{s} B \nabla v^{s} \cdot \nabla \psi_{0}-s \lambda^{2} a \nabla \psi_{0} \cdot \nabla \psi_{0} v^{2 s}\right] d x
\end{aligned}
$$

Упростим первые два слагаемых под знаком интеграла. Так как

$$
\lambda 2 a \nabla v^{s} \cdot \nabla \psi_{0} v^{s} \leqslant \lambda^{2} s a \nabla \psi_{0} \cdot \nabla \psi_{0} v^{2 s}+s^{-1} a \nabla v^{s} \cdot \nabla v^{s}
$$


и $(2 s-1) / s \geqslant 1$, то

$$
J \geqslant \int_{\mathbb{R}^{d}}\left(\nu \nabla v^{s} \cdot \nabla v^{s}-2 s \lambda B \nabla \psi_{0} \cdot \nabla v^{s} v^{s}-s^{2} \nu^{-1} \lambda^{2} v^{2 s}\right) d x .
$$

Используя оценку (2.3) для $s \lambda$ вместо $\lambda$, приходим к искомой оценке

$$
s\left(\mathscr{A}_{\psi} v, v^{2 s-1}\right)_{L^{2}} \geqslant \frac{\nu}{4}\left\|\nabla v^{s}\right\|_{2}^{2}-c_{1} g(s \lambda)\left\|v^{s}\right\|_{2}^{2}
$$

Нетрудно видеть, что неравенство (2.4) и, значит, оценка (2.7) справедливы при условии (1.12).

2.3. Теперь предположим, что выполнено условие (1.8), и возьмем $\psi(x)=$ $\lambda \psi_{0}(x), \psi_{0}(x)=|x|$. В данном случае $e^{\psi}=e^{\lambda|x|},\left|\nabla \psi_{0}\right| \leqslant 1$. Снова требуется оценить снизу квадратичную форму (2.2).

Лемма 2.2. Справедлива оченка (2.3), в которой постоянная $c_{0}$ зависит только от $d, \nu$ и величин $p$ u $l$ из (1.8).

ДокАзАтЕльство. Для единичного куба $\square$ положим

$$
\begin{aligned}
J & \equiv \lambda \int_{\square} B \nabla \psi_{0} \cdot \nabla v v d x \\
& =\lambda \int_{\square} B \nabla \psi_{0} \cdot \nabla v(v-m) d x+\lambda \int_{\square} B \nabla \psi_{0} \cdot \nabla v m d x=J_{1}+J_{2},
\end{aligned}
$$

где $m=\int_{\square} v d x$ - среднее значение. Используя прежние обозначения, по неравенству Гёльдера имеем

$$
\begin{aligned}
J_{1} & \leqslant|\lambda|\|B\|_{L^{p}(\square)}\|\nabla v\|_{L^{2}(\square)}\|v-m\|_{L^{q}(\square)} \\
& \leqslant c_{1}|\lambda|\|\nabla v\|_{L^{2}(\square)}\|\nabla v\|_{L^{2}(\square)}^{\theta}\|v-m\|_{2}^{1-\theta} \leqslant c_{2}|\lambda|\|\nabla v\|_{L^{2}(\square)}^{1+\theta}\|v\|_{L^{2}(\square)}^{1-\theta}
\end{aligned}
$$

в силу аналога мультипликативного неравенства (2.5) для единичного куба. Отсюда по неравенству Юнга

$$
J_{1} \leqslant c_{2} l|\lambda|\left(t^{2 /(1+\theta)}\|\nabla v\|_{L^{2}(\square)}^{2}+t^{-2 /(1-\theta)}\|v\|_{L^{2}(\square)}^{2}\right) \quad \forall t>0
$$

и, выбирая $t$ из соотношения $c_{2} l|\lambda| t^{2 /(1+\theta)}=\nu / 16$, получим

$$
J_{1} \leqslant \frac{\nu}{16}\|\nabla v\|_{L^{2}(\square)}^{2}+c_{1}|\lambda|^{\alpha}\|v\|_{L^{2}(\square)}^{2}, \quad \alpha=\frac{2}{1-\theta}=\frac{2 p}{p-d} .
$$

Слагаемое $J_{2}$ из (2.8) оценивается проще:

$$
\begin{aligned}
J_{2} & \leqslant \lambda \int_{\square} B \nabla \psi_{0} \cdot \nabla v d x \int_{\square} v d x \leqslant|\lambda|\|B\|_{L^{2}(\square)}\|\nabla v\|_{L^{2}(\square)}\|v\|_{L^{2}(\square)} \\
& \leqslant \frac{\nu}{16}\|\nabla v\|_{L^{2}(\square)}^{2}+c_{1}|\lambda|^{2}\|v\|_{L^{2}(\square)}^{2} .
\end{aligned}
$$

Теперь разобьем $\mathbb{R}^{d}$ на единичные кубы и, суммируя оценки (2.9), (2.10) по всем кубам, придем к искомой оценке (2.3). Лемма 2.2 доказана.

Из оценки (2.3) тем же способом, что и ранее, выводятся неравенства (2.6) и (2.7). 


\section{§3. Оценки для решения задачи Коши}

3.1. Решение задачи Коши (1.1) запишем как действие полугруппы:

$$
u(\cdot, t)=e^{-t \mathscr{A}} f .
$$

Рассмотрим также полугруппу $e^{-t \mathscr{A} \psi}$, которой соответствует задача Коши

$$
\frac{\partial v}{\partial t}=-\mathscr{A}_{\psi} v,\left.\quad v\right|_{t=0}=f
$$

Связь между этими полугруппами дается равенством

$$
e^{-t \mathscr{A}_{\psi}} f=e^{\psi} e^{-t \mathscr{A}} e^{-\psi} f
$$

которое справедливо по крайней мере для $f \in C_{0}^{\infty}\left(\mathbb{R}^{d}\right)$.

Умножая уравнение (3.1) скалярно на $v$, в силу (2.6) получим

$$
\begin{gathered}
\frac{1}{2} \frac{d}{d t}\|v\|_{2}^{2}=-\left(\mathscr{A}_{\psi} v, v\right)_{L^{2}} \leqslant \frac{c_{1}}{2} g(\lambda)\|v\|_{2}^{2}, \\
\int_{0}^{t}\|v\|_{2}^{2} d s \leqslant t e^{c_{1} t g(\lambda)}\|f\|_{2}^{2} .
\end{gathered}
$$

Можно считать, что начальное значение $f$ неотрицательно, и тогда решение $v(x, t)$ также неотрицательно. Умножим (3.1) скалярно на $s v^{2 s-1} \eta^{2}(t)$, где $\eta-$ гладкая неотрицательная функция на $[0, \infty), \eta(0)=0,0 \leqslant \eta(t) \leqslant 1$. В результате имеем

$$
\int_{\mathbb{R}^{d}} \eta^{2} \frac{\partial v}{\partial t} s v^{2 s-1} d x=\frac{1}{2} \frac{d}{d t} \int_{\mathbb{R}^{d}} \eta^{2} v^{2 s} d x-\int_{\mathbb{R}^{d}} \frac{d \eta}{d t} \eta v^{2 s} d x=-\eta^{2} s\left(\mathscr{A}_{\psi} v, v^{2 s-1}\right)_{L^{2}} .
$$

Отсюда с помощью (2.7) получаем оценку

$$
\sup _{0 \leqslant t \leqslant T} \int_{\mathbb{R}^{d}} \eta^{2} v^{2 s} d x+\int_{0}^{T} \int_{\mathbb{R}^{d}} \eta^{2}\left|\nabla v^{s}\right|^{2} d x d t \leqslant \widehat{c}\left[g(s \lambda)+\sup \left|\frac{\partial \eta}{\partial t}\right|\right] \int_{0}^{T} \int_{\mathbb{R}^{d}} \eta v^{2 s} d x d t .
$$

В мультипликативном неравенстве (2.5) возьмем специальный показатель $q=2(d+2) / d$. Тогда $\|u\|_{q}^{q} \leqslant c\|\nabla u\|_{2}^{2}\|u\|_{2}^{q-2}$, откуда

$$
\begin{aligned}
\left(\int_{t_{1}}^{t_{2}} \int_{\mathbb{R}^{d}}|u|^{q} d x d t\right)^{2 / q} & \leqslant c^{2 / q}\left(\int_{t_{1}}^{t_{2}}\|\nabla u\|_{2}^{2} d t\right)^{2 / q} \sup _{t_{1} \leqslant t \leqslant t_{2}}\|u\|_{2}^{2(q-2) / q} \\
& \leqslant c^{2 / q}\left\{\sup _{t_{1} \leqslant t \leqslant t_{2}}\|u\|_{2}^{2}+\int_{t_{1}}^{t_{2}}\|\nabla u\|_{2}^{2} d t\right\}
\end{aligned}
$$

где использовано неравенство Юнга $a^{2 / q} b^{2(q-2) / q} \leqslant a+b^{2}(a, b \geqslant 0)$.

Дальше применяем итерационную технику Мозера. С этой целью зафиксируем $t_{0}>0$ и рассмотрим интервалы

$$
I_{k}=t_{0}\left(\frac{1}{2}-\frac{1}{2^{k+1}}, 1\right), \quad k=0,1, \ldots
$$


а также гладкие функции $\eta_{k}=\eta_{k}(t), k=1,2, \ldots$, такие, что $0 \leqslant \eta_{k} \leqslant 1$ на $\left[0, t_{0}\right]$

$$
\eta_{k} \equiv 1 \quad \text { на } I_{k}, \quad \eta_{k} \equiv 0 \text { на } I_{0} \backslash I_{k-1}, \quad\left|\frac{d \eta_{k}}{d t}\right| \leqslant 4^{k} t_{0}^{-1} .
$$

Соединим неравенство (3.5) с неравенством (3.4), в котором $\eta$ заменено на $\eta_{k}$. Получим

$$
\begin{aligned}
\left(\int_{I_{k}} \int_{\mathbb{R}^{d}} v^{s q} d x d t\right)^{2 / q} & \leqslant c^{2 / q}\left(\sup _{t \in I_{k}}\left\|v^{s}\right\|_{2}^{2}+\int_{I_{k}}\left\|\nabla v^{s}\right\|_{2}^{2} d t\right) \\
& \leqslant c_{1}\left(g(s \lambda)+4^{k} t_{0}^{-1}\right) \int_{I_{k-1}} \int_{\mathbb{R}^{d}} v^{2 s} d x d t
\end{aligned}
$$

или, в других обозначениях,

$$
\left\|v^{s}\right\|_{L^{q}\left(\mathbb{R}^{d} \times I_{k}\right)} \leqslant c_{1}\left[g(s \lambda)+4^{k} t_{0}^{-1}\right]^{1 / 2}\left\|v^{s /(1+\gamma)}\right\|_{L^{q}\left(\mathbb{R}^{d} \times I_{k-1}\right)}^{1+\gamma}, \quad 1+\gamma=\frac{q}{2}=\frac{d+2}{d} .
$$

Положив

$$
s_{k}=(1+\gamma)^{k-1}, \quad \Phi_{k}=\left\|v^{s_{k}}\right\|_{L^{q}\left(\mathbb{R}^{d} \times I_{k}\right)},
$$

запишем (3.6) в виде рекуррентного соотношения

$$
\Phi_{k} \leqslant c_{1}\left[g\left(s_{k} \lambda\right)+4^{k} t_{0}^{-1}\right]^{1 / 2} \Phi_{k-1}^{1+\gamma}, \quad k=1,2, \ldots
$$

Заметим, что $g(s \lambda) \leqslant s^{\alpha} g(\lambda)$ (поскольку $\alpha>2, s \geqslant 1$ ) и $1+\gamma \leqslant 3$, так что

$$
\Phi_{k} \leqslant c_{1}\left[g(\lambda)+t_{0}^{-1}\right]^{1 / 2} 4^{k \alpha} \Phi_{k-1}^{1+\gamma} .
$$

Отсюда по индукции заключаем, что

$$
\Phi_{k} \leqslant c_{1}^{\left((1+\gamma)^{k}-1\right) / \gamma} 4^{\alpha\left((1+\gamma)^{k+1}-\gamma-1\right) / \gamma^{2}}\left[g(\lambda)+t_{0}^{-1}\right]^{\left((1+\gamma)^{k}-1\right) /(2 \gamma)} \Phi_{0}^{(1+\gamma)^{k}}
$$

и, следовательно,

$$
\sup _{\mathbb{R}^{d} \times\left[t_{0} / 2, t_{0}\right]} v \leqslant \lim _{k \rightarrow \infty} \sqrt[s_{k}]{\Phi_{k}} \leqslant c_{2}\left[g(\lambda)+t_{0}^{-1}\right]^{(1+\gamma) /(2 \gamma)} \Phi_{0}^{1+\gamma} .
$$

Поскольку $\Phi_{0}^{1+\gamma}=\|v\|_{L^{2}\left(\mathbb{R}^{d} \times\left[0, t_{0}\right]\right)}$, из (3.3) получаем неравенство

$$
\sup _{x \in \mathbb{R}^{d}}\left|v\left(x, t_{0}\right)\right| \leqslant c_{2}\left(g(\lambda)+t_{0}^{-1}\right)^{(1+\gamma) /(2 \gamma)} t_{0}^{1 / 2} e^{c_{1} t_{0} g(\lambda)}\|f\|_{2} .
$$

Заметим, что

$$
\left[g(\lambda)+t_{0}^{-1}\right]^{(1+\gamma) /(2 \gamma)} t_{0}^{1 / 2}=\left[t_{0} g(\lambda)+1\right]^{(1+\gamma) /(2 \gamma)} t_{0}^{-1 /(2 \gamma)} \leqslant c_{3} t_{0}^{-1 /(2 \gamma)} e^{c_{1} t_{0} g(\lambda)} .
$$

Отсюда (берем $t$ вместо $t_{0}$ )

$$
\left\|e^{-t \mathscr{A}_{\psi}} f\right\|_{\infty} \leqslant \frac{c}{t^{1 /(2 \gamma)}} e^{c_{1} t g(\lambda)}\|f\|_{2} .
$$


Аналогичная оценка таким же путем доказывается для сопряженного к $e^{-t \mathscr{A}} \psi$ оператора (который получится, если $\psi$ заменить на $-\psi$, а матрицу $B-$ на матрицу $-B)$. Поэтому выполнена двойственная к (3.7) оценка

$$
\left\|e^{-t \mathscr{A}_{\psi}} f\right\|_{2} \leqslant \frac{c}{t^{1 /(2 \gamma)}} e^{c_{1} t g(\lambda)}\|f\|_{1},
$$

которая вместе с самой оценкой (3.7) дает

$$
\left\|e^{-t \mathscr{A}_{\psi}} f\right\|_{\infty} \leqslant \frac{c_{0}}{t^{1 / \gamma}} e^{c_{0} t g(\lambda)}\|f\|_{1}, \quad \gamma=\frac{2}{d} .
$$

Полученное неравенство, в котором $f$ уже не обязательно неотрицательная функция, содержит в себе все утверждения теорем 1.1 и 1.2. В самом деле, непосредственно из (3.8) и соотношения (3.2) следует, что

$$
e^{\lambda \psi_{0}(x)}|v(x, t)| \leqslant \frac{c_{0}}{t^{d / 2}} e^{c_{0} t g(\lambda)} \int_{\mathbb{R}^{d}}|f(s)| d s,
$$

где $v(\cdot, t)=e^{-t \mathscr{A}} e^{-\psi} f$. Поэтому для решения задачи Коши (1.1) выполнена оценка (1.10).

Теперь несколько слов о фундаментальном решении. Из оценки (3.8) нетрудно вывести, что

$$
\sup _{x, y} K_{\psi}(x, y, t) \leqslant \frac{c_{0}}{t^{d / 2}} e^{c_{0} t g(\lambda)},
$$

где $K_{\psi}$ - ядро оператора $e^{-t \mathscr{A} \psi}$. Так как $K_{\psi}(x, 0, t)=e^{\lambda \psi_{0}(x)} K(x, 0, t)$, то

$$
K(x, 0, t) \leqslant \frac{c_{0}}{t^{d / 2}} e^{c_{0} t g(\lambda)-\lambda \psi_{0}(x)} .
$$

Если теперь в показателе взять инфимум по $\lambda$, то придем к оценкам (1.6), (1.9).

Эти же рассуждения можно было провести проще, если в оценке (1.10) непосредственно перейти к начальному значению $f(x)=\delta(x)$.

Отметим энергетические оценки, которые автоматически получаются из предыдущих результатов.

Для решения задачи Коши (1.1) имеем классическую оценку

$$
\|u(t, \cdot)\|_{2}^{2}+2 \nu \int_{0}^{t}\|\nabla u\|_{2}^{2} d s \leqslant\|f\|_{2}^{2} .
$$

Для решения задачи Коши (3.1) выполнено

$$
\begin{gathered}
\frac{1}{2} \frac{d}{d t}\|v\|_{2}^{2}+\left(\mathscr{A}_{\psi} v, v\right)=0 \\
\frac{1}{2} \frac{d}{d t}\|v\|_{2}^{2}+\frac{\nu}{2}\|\nabla v\|_{2}^{2} \leqslant \frac{c_{1}}{2} g(\lambda)\|v\|_{2}^{2}
\end{gathered}
$$

в силу оценки (2.6). Отсюда получим

$$
\|v(t, \cdot)\|_{2}^{2}+\nu \int_{0}^{t}\|\nabla v\|_{2}^{2} d s \leqslant\|f\|_{2}^{2} e^{c_{1} t g(\lambda)} .
$$


Для решения исходной задачи (1.1) эта оценка дает

$$
\left\|e^{\psi} u(\cdot, t)\right\|_{2}^{2}+\nu \int_{0}^{t}\left\|\nabla\left(e^{\psi} u\right)\right\|_{2}^{2} d s \leqslant\left\|e^{\psi} f\right\|_{2}^{2} e^{c_{1} t g(\lambda)},
$$

где $\psi=\lambda \psi_{0}$. Так как $\left|\nabla \psi_{0}\right| \leqslant 1$ и

$$
\begin{gathered}
\left\|\nabla\left(e^{\psi} u\right)\right\|_{2}^{2} \geqslant \frac{1}{2}\left\|e^{\psi} \nabla u\right\|_{2}^{2}-2 \lambda^{2}\left\|e^{\psi} u\right\|_{2}^{2}, \\
\int_{0}^{t}\left\|e^{\psi} u\right\|_{2}^{2} d s \leqslant t\left\|e^{\psi} f\right\|_{2}^{2} e^{c_{1} t g(\lambda)},
\end{gathered}
$$

то из (3.10) следует оценка (1.11).

3.2. Выведем из оценки (1.5) одно важное для дальнейшего свойство решений.

Лемма 3.1. Пусть $A_{\varepsilon}(x)$ - семейство матрич, для которых условия (1.2), (1.4) выполнены равномерно по $\varepsilon, u_{\varepsilon}$ - аппроксимачионное решение задачи Коши с матрицей $A_{\varepsilon}(x)$ и дельтаобразным начальным значением

$$
u_{\varepsilon}(x, 0)=f_{\varepsilon}(x)=\varepsilon^{-d} f\left(\varepsilon^{-1} x\right),
$$

где $f$ удовлетворяет условиям

$$
f \geqslant 0, \quad\left(x^{2}+1\right)^{\lambda} f \in L^{1}\left(\mathbb{R}^{d}\right) \quad \forall \lambda>0, \quad \int_{\mathbb{R}^{d}} f d x=1 .
$$

Тогда

$$
\lim _{\varepsilon \rightarrow 0} \int_{|x| \geqslant r} u_{\varepsilon}(x, t) d x \leqslant c_{r}(t), \quad \lim _{t \rightarrow 0} c_{r}(t)=0 \quad \forall r>0,
$$

где Функиия $c_{r}(t)$ определяется толъко величинами $d, \nu, p, l$.

ДоказАТЕЛЬСтво. Из (1.5) имеем

$$
u_{\varepsilon}(x, t) \leqslant \frac{c_{0} e^{c_{0} t g(\lambda)}}{\left(x^{2}+1\right)^{\lambda} t^{d / 2}} \int_{\mathbb{R}^{d}}\left(\varepsilon^{2} y^{2}+1\right)^{\lambda} f(y) d y .
$$

Отсюда

$$
\begin{aligned}
c_{r}(t) & \equiv \lim _{\varepsilon \rightarrow 0} \int_{|x| \geqslant r} u_{\varepsilon} d x \leqslant \frac{c_{0}}{t^{d / 2}} e^{c_{0} t g(\lambda)} J(r) \lim _{\varepsilon \rightarrow 0} \int_{\mathbb{R}^{d}}\left(\varepsilon^{2} y^{2}+1\right)^{\lambda} f(y) d y \\
& =\frac{c_{0}}{t^{d / 2}} e^{c_{0} t g(\lambda)} J(r), \\
J(r) & =\int_{|x| \geqslant r} \frac{d x}{\left(x^{2}+1\right)^{\lambda}} .
\end{aligned}
$$

Поскольку при больших $\lambda>0$

$$
J(r) \leqslant \frac{c_{1}(r)}{\left(r^{2}+1\right)^{\lambda}},
$$

то, положив $\lambda^{\alpha}=t^{-1}$, получим $t g(\lambda) \leqslant 2 t \lambda^{\alpha}=2$,

$$
c_{r}(t) \leqslant \frac{c_{0} c_{1} e^{2 c_{0}}}{t^{d / 2}\left(r^{2}+1\right)^{1 / t^{1 / \alpha}}} \rightarrow 0 \quad \text { при } t \rightarrow 0 .
$$

Лемма 3.1 доказана. 
Доказанная лемма теряет силу, если ослабить условие (1.4), положив в нем $p<d$. Рассмотрим следующий пример. Пусть $d=2$,

$$
A(x)=\left(\begin{array}{cc}
1 & \beta\left(x_{1}\right) \\
-\beta\left(x_{1}\right) & 1
\end{array}\right),
$$

где $\beta\left(x_{1}\right)$ - гладкая периодическая (с периодом 1$)$ функция. Если $K_{\varepsilon}(x, 0, t)-$ фундаментальное решение для задачи Коши с $A_{\varepsilon}(x)=A\left(\varepsilon^{-1} x\right)$, то по теории усреднения имеет место поточечная сходимость

$$
K_{\varepsilon}(x, 0, t) \rightarrow K_{0}(x, t), \quad x \in \mathbb{R}^{d}, \quad t>0,
$$

где $K_{0}(x, t)$ - фундаментальное решение для усредненной задачи Коши

$$
\frac{\partial u}{\partial t}=\operatorname{div} A_{0} \nabla u,\left.\quad u\right|_{t=0}=\delta(x),
$$

$A_{0}$ - усредненная матрица. Матрица $A_{0}$ в данном случае (см. [3; гл. I, §2]) вычисляется точно:

$$
A_{0}=\left(\begin{array}{cc}
1 & 0 \\
0 & a^{2}
\end{array}\right), \quad a^{2}=1+\left\langle\beta^{2}\right\rangle, \quad\left\langle\beta^{2}\right\rangle=\int_{0}^{1} \beta^{2}\left(x_{1}\right) d x_{1} .
$$

Кроме того, для $K_{\varepsilon}$ выполнена равномерная по $\varepsilon$ оценка Нэша-Аронсона (1.3), из которой получаем, что

$$
\int_{|x| \geqslant r} K_{0}(x, t) d x=\lim _{\varepsilon \rightarrow 0} \int_{|x| \geqslant r} K_{\varepsilon}(x, 0, t) d x .
$$

Допустим теперь, что имеет место свойство (1.7), в котором функция $c_{r}(t)$ определяется только константой $l=\left\langle|\beta|^{p}\right\rangle$ при некотором $p<2$, т.е.

$$
\int_{|x|>r} K_{0}(x, t) d x \leqslant c_{r}(t), \quad \lim _{t \rightarrow 0} c_{r}(t)=0 \quad \forall r>0 .
$$

Но при условии $\left\langle|\beta|^{p}\right\rangle \leqslant l$ величина $\left\langle\beta^{2}\right\rangle$ может быть сделана сколь угодно большой. Получается, что свойство (3.13) выполняется для

$$
K_{0}(x, t)=\frac{1}{2 \pi t a} e^{-x_{1}^{2} /(4 t)} e^{-x_{2}^{2} /\left(4 a^{2} t\right)}
$$

равномерно по $a \geqslant 1$. Легко понять, что это невозможно.

3.3. Нам понадобится также одно свойство компактности для семейства задач Коши.

Лемма 3.2. Пусть $A^{\varepsilon}(x)$ - семейство матрии, для которых условия (1.2), (1.4) выполнены равномерно по $\varepsilon, u_{\varepsilon}(x, t)$ - аппроксимачионное решение задачи Коши с начальным значением $u_{\varepsilon}(x, 0)=f_{\varepsilon}(x)$, удовлетворяющим условиям:

(a) семейство $f_{\varepsilon}$ ограничено в $L^{2}\left(\mathbb{R}^{d}\right)$;

(b) для любого $\delta>0$ найдется $R=R(\delta)$ такое, что

$$
\int_{|x| \geqslant R}\left|f_{\varepsilon}\right|^{2} d x \leqslant \delta
$$

Тогда семейство $u_{\varepsilon}(x, t)$ компактно в $L^{2}\left(\mathbb{R}^{d} \times[0, T]\right)$. 
Доказательство разобьем на отдельные замечания.

(i) Благодаря условию (b) и оценке (3.9) можно считать, что семейство начальных значений $f_{\varepsilon}$ финитно, т.е. $f_{\varepsilon}$ сосредоточены в одном и том же шаре.

(ii) Тогда энергетическая оценка (1.11) действует при любом $\lambda>0$, и из нее ясно, что достаточно доказать компактность семейства $u_{\varepsilon}(x, t)$ в $L^{2}(Q \times[0, T])$, где $Q$ - любая ограниченная область.

(iii) Из оценки (3.9) следует, что семейство $u_{\varepsilon}$ ограничено в пространстве $C\left(0, T, L^{2}(Q)\right)$ непрерывных функций со значениями в $L^{2}(Q)$. Проверим, что это семейство слабо равностепенно непрерывно. Достаточно убедиться, что для любой $\varphi \in C_{0}^{\infty}\left(\mathbb{R}^{d}\right)$ семейство функций

$$
\Phi_{\varepsilon}(t)=\int_{\mathbb{R}^{d}} u_{\varepsilon}(x, t) \varphi(x) d x
$$

равностепенно непрерывно на $[0, T]$. Исходим из равенства

$$
\Phi_{\varepsilon}\left(t_{2}\right)-\Phi_{\varepsilon}\left(t_{1}\right)=\int_{t_{1}}^{t_{2}} \int_{\mathbb{R}^{d}} A^{\varepsilon} \nabla u_{\varepsilon} \cdot \nabla \varphi d x,
$$

которое очевидно в случае ограниченной матрицы $A^{\varepsilon}$ и легко проверяется для аппроксимационного решения. Отсюда

$$
\begin{aligned}
& \left|\Phi_{\varepsilon}\left(t_{2}\right)-\Phi_{\varepsilon}\left(t_{1}\right)\right| \leqslant\left|\int_{t_{1}}^{t_{2}} \int_{\mathbb{R}^{d}} A^{\varepsilon} \nabla u_{\varepsilon} \cdot \nabla \varphi d x d t\right| \\
& \quad \leqslant\left|t_{1}-t_{2}\right|^{1 / 2} \sup |\nabla \varphi|\left(\int_{Q}\left|A^{\varepsilon}\right|^{2} d x\right)^{1 / 2}\left(\int_{t_{1}}^{t_{2}} \int_{\mathbb{R}^{d}}\left|\nabla u_{\varepsilon}\right|^{2} d x d t\right) \leqslant C\left|t_{1}-t_{2}\right|^{1 / 2},
\end{aligned}
$$

где $C$ не зависит от $\varepsilon$.

(iv) Теперь достаточно ссылки на известную лемму о компактности (см. [16; гл. IX]). Сформулируем эту лемму применительно к нашим функциональным пространствам.

Лемма 3.3. Пусть семейство $z_{\varepsilon}=z_{\varepsilon}(x, t)$ ограничено в пространствах $C\left(0, T, L^{2}(Q)\right)$ и $L^{2}\left(0, T, H^{1}(Q)\right)$, а также слабо равностепенно непрерывно в $L^{2}(Q)$. Тогда это семейство компактно в $L^{2}\left(0, T, L^{2}(Q)\right)$.

Для удобства чтения приведем доказательство леммы 3.3. Рассмотрим конечномерные проекторы

$$
P_{n}: L^{2}(Q) \rightarrow L^{2}(Q),
$$

удовлетворяющие условию $P_{n} f \rightarrow f$ в $L^{2}(Q)$ для любого $f \in L^{2}(Q)$. Сильная сходимость операторов всегда равномерна на компактах, поэтому для любого $\delta>0$

$$
\left\|P_{n} f-f\right\|_{L^{2}(Q)}^{2} \leqslant \delta\|f\|_{H^{1}(Q)}^{2}
$$

при $n \geqslant n(\delta)$. Положив $z_{\varepsilon}^{n}=P_{n} z_{\varepsilon}$, получим

$$
\int_{0}^{T}\left\|z_{\varepsilon}^{n}-z_{\varepsilon}\right\|_{L^{2}(Q)}^{2} d t \leqslant \delta\left\|z_{\varepsilon}\right\|_{L^{2}\left(0, T, H^{1}(Q)\right)}^{2} \leqslant c \delta .
$$

При фиксированном $n$ семейство $z_{\varepsilon}^{n}$ компактно в $L^{2}\left(0, T, L^{2}(Q)\right)$ в силу слабой равностепенной непрерывности исходного семейства $z_{\varepsilon}$. Тогда утверждение 
леммы следует из (3.14) по критерию компактности Хаусдорфа (множество компактно тогда и только тогда, когда для любого $\delta>0$ найдется компактная $\delta$-сеть).

\section{§ 4. Существование аппроксимационной полугруппы}

4.1. Нашей целью будет доказательство теоремы 1.3 о существовании аппроксимационной полугруппы.

Напомним, что если матрица $B$ ограничена, то оператор $\mathscr{A}=-\operatorname{div} A \nabla$ обладает следующими свойствами:

(i) область определения $D(\mathscr{A})$ плотна в $L^{2}\left(\mathbb{R}^{d}\right)$;

(ii) отрицательная полуось принадлежит резольвентному множеству и $\left\|(\mathscr{A}+s)^{-1}\right\| \leqslant s^{-1}$.

Здесь и далее через " 1 " обозначаем тождественный оператор.

Пусть для матрицы $B$ выполнено только условие (1.17). Рассмотрим аппроксимации $B_{n}$ из (1.18) и соответствующие операторы $\mathscr{A}_{n}$.

Важную роль будет играть следующая

ЛЕмма 4.1 (лемма о компактности). Для любого $f \in L^{2}\left(\mathbb{R}^{d}\right)$ последовательность $\left(\mathscr{A}_{n}+1\right)^{-1} f$ компактна в смысле сильной сходимости в $L^{2}\left(\mathbb{R}^{d}\right)$.

Эту лемму докажем позже.

Теорема 4.2. Последовательность $\mathscr{A}_{n}$ компактна в смысле сильной резольвентной сходимости. Другими словами, с точностью до выделения подпоследовательности выполнено

$$
\left(\mathscr{A}_{n}+s\right)^{-1} f \stackrel{L^{2}\left(\mathbb{R}^{d}\right)}{\longrightarrow}(\mathscr{A}+s)^{-1} f \quad \forall s>0, \quad f \in L^{2}\left(\mathbb{R}^{d}\right),
$$

где $\mathscr{A}$ - оператор, удовлетворяющий условиям (i), (ii).

ДокАЗАтельство. Рассмотрим сначала случай $s=1$. На основе леммы 4.1 с помощью диагонального метода убеждаемся в том, что последовательность $\left(\mathscr{A}_{n}+1\right)^{-1}$ компактна в смысле сильной сходимости операторов. Можно считать, что она сама является сходящейся, т.е.

$$
\left(\mathscr{A}_{n}+1\right)^{-1} f \rightarrow S f \quad \forall f \in L^{2}\left(\mathbb{R}^{d}\right) .
$$

Проверим, что область значений оператора $S$ плотна в $L^{2}\left(\mathbb{R}^{d}\right)$. Сильная сходимость (4.2) влечет слабую сходимость сопряженных операторов:

$$
\left(\mathscr{A}_{n}^{*}+1\right)^{-1} g \rightarrow S^{*} g \quad \forall g \in L^{2}\left(\mathbb{R}^{d}\right) .
$$

Если допустить, что область значений $S$ не плотна в $L^{2}\left(\mathbb{R}^{d}\right)$, то оператор $S^{*}$ имеет непустое ядро, т.е. $v=S^{*} g=0$ для некоторого $g \in L^{2}\left(\mathbb{R}^{d}\right), g \neq 0$. Но $v$ есть решение эллиптического уравнения

$$
-\operatorname{div}(a \nabla v-B \nabla v)+v=g,
$$

и тогда ясно, что $g=0$. Итак, область значений оператора $S$ плотна в $L^{2}\left(\mathbb{R}^{d}\right)$. Положив $S^{-1}=\mathscr{A}+1$, получим соотношение (4.1) при $s=1$. Как показано в книге Като [17; гл. VIII, теорема 1.3], отсюда следует выполнение соотношения (4.1) при любом $s>0$. Теорема 4.2 доказана.

Теперь, чтобы получить теорему 1.3 , достаточно ссылки на теорему Троттера-Като [17; гл. IX, теорема 2.16]. 
4.2. Для доказательства леммы о компактности (леммы 4.1) нам понадобится следующее утверждение.

Лемма 4.3. Пусть $B \in L^{\infty}\left(\mathbb{R}^{d}\right), u$-решение уравнения $(1.13), f \in C_{0}^{\infty}\left(\mathbb{R}^{d}\right)$. Тогда при $d \geqslant 3$ справедлива ощенка

$$
\int_{\mathbb{R}^{d}} \ln ^{2 \lambda}\left(x^{2}+e\right) u^{2}(x) d x \leqslant c_{0} \int_{\mathbb{R}^{d}} \ln ^{2 \lambda}\left(x^{2}+e\right) f^{2}(x) d x,
$$

в которой показатель $\lambda>0$ достаточно мал, а постоянная с 0 зависит только от $d, \nu$ и величины $l$ из (1.17).

ДокАзАтельство. Сначала докажем эту оценку для решения задачи Дирихле в кубе $Q=[-n, n]^{d}$. Итак, пусть

$$
u \in H_{0}^{1}(Q), \quad-\operatorname{div}(a \nabla u+B \nabla u)+u=f .
$$

Возьмем $\psi=\lambda \psi_{0}, \psi_{0}=\ln \ln \left(x^{2}+e\right), \lambda>0$, и рассмотрим оператор $\mathscr{A}_{\psi}=$ $e^{\psi} \mathscr{A} e^{-\psi}$. Для $v=e^{\psi} u$ выполнено

$$
\begin{gathered}
v \in H_{0}^{1}(Q), \quad \mathscr{A}_{\psi} v+v=g=e^{\psi} f \\
\int_{Q} a \nabla v \cdot \nabla v d x+2 \lambda \int_{Q} B \nabla v \cdot \nabla \psi_{0} v d x \\
-\lambda^{2} \int_{Q} a \nabla \psi_{0} \cdot \nabla \psi_{0} v^{2} d x+\int_{Q} v^{2} d x=\int_{Q} g v d x .
\end{gathered}
$$

Заметим, что

$$
\left|\nabla \psi_{0}\right| \leqslant \frac{2|x|}{\left(x^{2}+1\right) \ln \left(x^{2}+e\right)}
$$

и $|B|\left|\nabla \psi_{0}\right| \in L^{d}\left(\mathbb{R}^{d}\right)$. Последнее проверяется путем перехода к сферическим координатам и интегрирования по частям (см. доказательство неравенства (2.4)). Отсюда по неравенству Гёльдера и Соболева

$$
\begin{aligned}
\left|\int_{Q} B \nabla v \cdot \nabla \psi_{0} v d x\right| & \leqslant\left(\int_{Q}|B|^{d}\left|\nabla \psi_{0}\right|^{d} d x\right)^{\frac{1}{d}}\left(\int_{Q}|\nabla v|^{2} d x\right)^{\frac{1}{2}}\left(\int_{Q}|v|^{\frac{2 d}{d-2}} d x\right)^{\frac{d-2}{2 d}} \\
& \leqslant c_{1} \int_{Q}|\nabla v|^{2} d x
\end{aligned}
$$

Тогда из (4.4) при малых $\lambda$ следует оценка

$$
\int_{Q} v^{2} d x \leqslant c_{2} \int_{Q} g^{2} d x, \quad v=e^{\psi} u, \quad g=e^{\psi} f
$$

в которой постоянная $c_{2}$ не зависит от $n$. Собственно оценка (4.3) получается предельным переходом при $n \rightarrow \infty$. Лемма 4.3 доказана.

Проверим, что в случае $d=2$ верна оценка

$$
\int_{\mathbb{R}^{d}} \ln ^{2 \lambda}\left(x^{2}+e\right) u^{2}(x) d x \leqslant c_{0}\left\{\int_{\mathbb{R}^{d}} \ln ^{2 \lambda}\left(x^{2}+e\right) f^{2}(x) d x+\|f\|_{\infty}^{2}\right\} .
$$


Действительно, так как по принципу максимума $\|u\|_{\infty} \leqslant\|f\|_{\infty}=M$, то $|v| \leqslant$ $e^{\psi}\|f\|_{\infty}=M \ln ^{\lambda}\left(x^{2}+e\right)$. Легко выясняется, что при малых $\lambda>0$

$$
|B|\left|\nabla \psi_{0}\right||v| \leqslant \frac{2 M|B||x|}{\left(x^{2}+e\right) \ln ^{1-\lambda}\left(x^{2}+e\right)} \in L^{2}\left(\mathbb{R}^{2}\right) .
$$

Отсюда следует неравенство

$$
\left|\int_{Q} B \nabla v \cdot \nabla \psi_{0} v d x\right| \leqslant c\left(\int_{Q}|\nabla v|^{2} d x\right)^{1 / 2} M
$$

и сама оценка (4.5).

Теперь не составляет труда доказать собственно лемму о компактности. Так как $\left\|\left(\mathscr{A}_{n}+1\right)^{-1}\right\| \leqslant 1$, то достаточно установить компактность последовательности $\left(\mathscr{A}_{n}+1\right)^{-1} f$ для $f$ из плотного в $L^{2}\left(\mathbb{R}^{d}\right)$ множества. Но для $f \in C_{0}^{\infty}\left(\mathbb{R}^{d}\right)$ компактность следует из энергетической оценки (1.16) и оценки (4.3) (в двумерном случае - из оценки (4.5)).

\section{§ 5. Достаточные условия единственности}

По теореме 1.3 задаче Коши (1.1) отвечает хотя бы одна аппроксимационная полугруппа. Чтобы обеспечить единственность такой полугруппы, требуются определенные локальные условия на матрицу $B$. Можно указать несколько таких условий - все они заимствуются из найденных в [4] условий единственности задачи Дирихле в ограниченной области. Остановимся на одном из локальных условий.

ТеОРема 5.1. Пусть выполнено условие (1.17) и, кроме того,

$$
b=\operatorname{div} B \in L_{\mathrm{loc}}^{2}\left(\mathbb{R}^{d}\right) .
$$

Тогда аппроксимачионная полугруппа единственна.

Теорема 5.1 будет получена в качестве следствия подходящих свойств единственности для резольвентного эллиптического уравнения вида (1.13). Предварительно отметим одно утверждение.

Лемма 5.2. Пусть $u \in H^{1}\left(\mathbb{R}^{d}\right), \bar{u}-$ срезка,

$$
\bar{u}=\left\{\begin{array}{rr}
u, & \text { если }|u| \leqslant N \\
\pm N, & \text { если }|u|>N
\end{array}\right.
$$

Тогда

$$
\int_{\mathbb{R}^{d}} b \cdot \nabla u \bar{u} \varphi d x=-\int_{\mathbb{R}^{d}} B \nabla u \cdot \nabla \varphi \bar{u} d x \quad \forall \varphi \in C_{0}^{\infty}\left(\mathbb{R}^{d}\right) .
$$

ДоказАТЕЛЬСтво. Тождество (1.20) запишем коротко в виде

$$
\int_{\mathbb{R}^{d}} b \cdot h d x=-\int_{\mathbb{R}^{d}} B \cdot \nabla h d x \quad \forall h \in C_{0}^{\infty}\left(\mathbb{R}^{d}\right)^{d} .
$$


Считая пока функцию $u$ гладкой, возьмем в этом тождестве пробный вектор $h=\nabla u \bar{u} \varphi$. Так как в этом случае

$$
\begin{gathered}
\nabla h=\varphi(\nabla u \times \nabla \bar{u})+\bar{u}(\nabla u \times \nabla \varphi)+\bar{u} \varphi \nabla^{2} u, \\
B \nabla u \cdot \nabla \bar{u}=0, \quad B \cdot \nabla^{2} u=0,
\end{gathered}
$$

то равенство (5.2) имеет место. Путем аппроксимации оно распространяется на функции $u \in H^{1}\left(\mathbb{R}^{d}\right)$. Лемма 5.2 доказана.

Основной шаг составляет следующий результат о единственности для эллиптического уравнения.

ТеОрема 5.3. Пусть выполнены условия (1.17), (5.1). Тогда при $d \geqslant 3$ решение эллиптического уравнения (1.13) единственно, а при $d=2$ аппроксимационное решение этого уравнения единственно.

ДокАзАтЕльство. Допустим, что однородное уравнение имеет решение, т.е.

$$
u \in H^{1}\left(\mathbb{R}^{d}\right), \quad \int_{\mathbb{R}^{d}}(a \nabla u \cdot \nabla h-b \cdot \nabla u h+u h) d x=0 \quad \forall h \in C_{0}^{\infty}\left(\mathbb{R}^{d}\right) .
$$

В качестве пробной функции $h$ в действительности можно взять

$$
h=\bar{u} \varphi, \quad \varphi \in C_{0}^{\infty}\left(\mathbb{R}^{d}\right),
$$

в чем можно убедиться путем аппроксимации, если учесть, что $b \cdot \nabla u \in L_{\text {loc }}^{1}\left(\mathbb{R}^{d}\right)$. По лемме 5.2

$$
\int_{\mathbb{R}^{d}}(a \nabla u \cdot \nabla \bar{u}+u \bar{u}) \varphi d x=-\int_{\mathbb{R}^{d}} a \nabla u \cdot \nabla \varphi \bar{u} d x+\int_{\mathbb{R}^{d}} B \nabla u \cdot \nabla \varphi \bar{u} d x .
$$

Считаем, что $\varphi \geqslant 0$ зависит от параметра $r$,

$$
\varphi=\left\{\begin{array}{ll}
1, & \text { если }|x| \leqslant r / 2, \\
0, & \text { если }|x| \geqslant r,
\end{array} \quad|\nabla \varphi| \leqslant \frac{4}{r} .\right.
$$

Покажем, что второй интеграл в правой части (5.3) стремится к нулю при $r \rightarrow \infty$. Для $d \geqslant 3$ по неравенствам Гёльдера и Соболева

$$
\begin{aligned}
J= & \left|\int_{\mathbb{R}^{d}} B \nabla u \cdot \nabla \varphi \bar{u} d x\right| \\
\leqslant & \frac{2}{r}\left(\int_{|x| \leqslant r}|B|^{d} d x\right)^{1 / d}\left(\int_{|x| \geqslant r / 2}|\nabla u|^{2} d x\right)^{1 / 2}\left(\int_{\mathbb{R}^{d}}|\bar{u}|^{2 d /(d-2)} d x\right)^{(d-2) /(2 d)} \\
& \quad \int_{\mathbb{R}^{d}}|\bar{u}|^{2 d /(d-2)} d x \leqslant \int_{\mathbb{R}^{d}}|u|^{2 d /(d-2)} d x \leqslant c_{0}\left(\int_{\mathbb{R}^{d}}|\nabla u|^{2} d x\right)^{d /(d-2)}
\end{aligned}
$$

Отсюда и из (1.17) следует, что $J \rightarrow 0$ при $r \rightarrow \infty$. Сходимость к нулю первого слагаемого в правой части (5.3) устанавливается аналогично (и даже проще). В результате приходим к равенству

$$
\int_{\mathbb{R}^{d}}(a \nabla u \cdot \nabla \bar{u}+u \bar{u}) d x=0
$$

и, полагая в срезке $N \rightarrow \infty$, получим, что $u=0$. 
В случае $d=2$ изложенное доказательство единственности годится только при условии (1.4) вместо (1.17). При условии (1.17) требуется отдельное рассмотрение. Покажем, что однородное уравнение не имеет ненулевых решений из $H^{1}\left(\mathbb{R}^{d}\right) \cap L^{\infty}\left(\mathbb{R}^{d}\right)$. Как показано в [4; лемма 1.5$]$, отсюда следует единственность аппроксимационного решения. В данном случае нет необходимости брать срезку и вместо $\bar{u}$ в (5.3) можно взять $u$. Благодаря ограниченности $u$ сходимость к нулю правой части при $r \rightarrow \infty$ устанавливается непосредственно по неравенству Гёльдера. Теорема 5.3 доказана.

\section{§ 6. Диффузия в несжимаемом случайном потоке. Центральная предельная теорема}

6.1. Пусть $\Omega$ - вероятностное пространство, т.е. множество со счетно аддитивной нормированной мерой $\mu$, заданной на некоторой $\sigma$-алгебре $\mathscr{F}$ его подмножеств.

Динамической системой $c d$-мерным временем или просто $d$-мерной динамической системой называется такое семейство отображений $T(x): \Omega \rightarrow \Omega$ $\left(x \in \mathbb{R}^{d}\right)$, что выполнены условия:

1) групповое свойство: $T(0)=I$ (тождественное отображение),

$$
T(x+y)=T(x) T(y) \quad \forall x, y \in \mathbb{R}^{d} ;
$$

2) преобразования $T(x): \Omega \rightarrow \Omega$ сохраняют меру $\mu$ на $\Omega$, т.е. для любых $x \in \mathbb{R}^{d}, F \in \mathscr{F}$

$$
T(x) F \in \mathscr{F}, \quad \mu(T(x) F)=\mu(F) ;
$$

$3)$ для любой $\mu$-измеримой функции $h(\omega)$ на $\Omega$ функция $h(T(x) \omega)$ измерима на $\Omega \times \mathbb{R}^{d}$ (при этом на $\mathbb{R}^{d}$ задается мера Лебега).

Измеримая на $\Omega$ функция $h$ называется инвариантной, если $h(T(x) \omega)=h(\omega)$ для любого $x \in \mathbb{R}^{d} \mu$-п.в. Динамическая система эргодична, если всякая инвариантная функция есть константа ( $\mu$-п.в.). В этой ситуации говорят также, что мера $\mu$ эргодична относительно $T(x)$. Мы предполагаем, что динамическая система $T(x)$ эргодична, и используем эргодическую теорему в следующей форме.

ЭРГОДИЧЕСКАЯ ТЕОРЕМА [18; гл. VIII, 7]. Пусть $h \in L^{q}(\Omega)=L^{q}(\Omega, d \mu)$, $q \geqslant 1$. Тогда для почти всех $\omega \in \Omega$ реализащия $h(T(x) \omega)$ принадлежит $L_{\mathrm{loc}}^{q}\left(\mathbb{R}^{d}\right)$ $u$ npu $\varepsilon \rightarrow 0$

$$
h\left(T\left(\varepsilon^{-1} x\right) \omega\right) \rightarrow\langle h\rangle=\int_{\Omega} h d \mu \quad \text { слабо в } L_{\mathrm{loc}}^{q}\left(\mathbb{R}^{d}\right) .
$$

Для большинства приложений достаточно считать, что $\Omega$ - компактное метрическое пространство, $\mu$ - борелевская мера, а преобразование

$$
\Omega \times \mathbb{R}^{d} \rightarrow \Omega, \quad \text { где }(\omega, x) \rightarrow T(x) \omega,
$$

непрерывно. Приведем простейшие примеры.

ПримеР 1 (периодический случай). Пусть $\Omega=\square$ - единичный куб,

$$
\square=\left\{\omega \in \mathbb{R}^{d}, 0 \leqslant \omega_{j} \leqslant 1, j=1, \ldots, d\right\} .
$$


На $\Omega$ имеется динамическая система $T(x) \omega=\omega+x(\bmod 1)$, относительно которой мера Лебега инвариантна и эргодична. Реализация функции $h(\omega)$ имеет вид $h(x+\omega)$.

ПримеР 2 (квазипериодический случай). Пусть $\Omega=\square$ - единичный куб в $\mathbb{R}^{m}, \mu$ - мера Лебега на нем. Для $x \in \mathbb{R}^{d}$ положим $T(x) \omega=\omega+\lambda x(\bmod 1)$, где $\lambda=\left\{\lambda_{i j}\right\}$ - матрица размера $d \times m$. Очевидно, преобразование $T(x)$ сохраняет меру $\mu$ на $\Omega$. Эргодичность будет иметь место, если $\lambda_{i j} k_{j} \neq 0$ для любого целочисленного вектора $k$. Таким образом, $L^{q}(\Omega)=L^{q}(\square)-$ пространство периодических функций от $m$ переменных, а реализации имеют вид $h(\omega+\lambda x)$.

6.2. Пусть $A=A(\omega)-\mu$-измеримая эллиптическая матрица,

$$
\nu \xi^{2} \leqslant A(\omega) \xi \cdot \xi \leqslant \nu^{-1} \xi^{2},
$$

где $\nu>0$ не зависит от $\omega$. Условия на кососимметрическую часть $B$ этой матрицы будут указаны позже, а сейчас напомним постановку вопроса о центральной предельной теореме в классическом случае $B \in L^{\infty}(\Omega)$.

Рассмотрим задачу Коши (1.1), в которой $A(x)=A(T(x) \omega)$, а начальное значение $f$ взято не зависящим от $\omega$,

$$
f \geqslant 0, \quad f \in L^{1}\left(\mathbb{R}^{d}\right), \quad \int_{\mathbb{R}^{d}} f d x=1,
$$

и пусть $X(t)=X(t, \omega)$ - случайный процесс с плотностью распределения $u(x, t, \omega)$. Центральная предельная теорема - это утверждение о том, что при $\varepsilon \rightarrow 0$ случайная величина $\varepsilon X\left(\varepsilon^{-2} t\right)(t>0)$ сходится по распределению к гауссовой случайной величине. Очевидно, что плотностью распределения указанной случайной величины служит $\varepsilon^{-d} u\left(\varepsilon^{-1} x, \varepsilon^{-2} t, \omega\right)-$ решение задачи Коши для уравнения с матрицей $A\left(\varepsilon^{-1} x\right)$ и дельтаобразным начальным значением $\varepsilon^{-d} f\left(\varepsilon^{-1} x\right)$. Более точно, сходимость по распределению и сама центральная предельная теорема означают, что для $\mu$-почти всех $\omega$

$$
\lim _{\varepsilon \rightarrow 0} \int_{\mathbb{R}^{d}}\left[\varepsilon^{-d} u\left(\varepsilon^{-1} x, \varepsilon^{-2} t, \omega\right)-K_{0}(x, t)\right] \varphi(x) d x=0 \quad \forall t \geqslant 0, \quad \varphi \in C_{0}^{\infty}\left(\mathbb{R}^{d}\right),
$$

где

$$
K_{0}(x, t)=(2 \pi t)^{-d / 2}\left(\operatorname{det} A_{0}\right)^{-1 / 2} e^{-A_{0}^{-1} x \cdot x /(4 t)},
$$

$A_{0}$ - постоянная положительно определенная матрица, называемая матрицей эффективной диффузии.

Укажем эллиптическую задачу на вероятностном пространстве $\Omega$, с помощью которой определяется матрица $A_{0}$. Напомним, что вектор $v=v(\omega)$, заданный на $\Omega, v \in L^{1}(\Omega)^{d}$, потенциален (соленоидален), если для $\mu$-почти всех $\omega \in \Omega$ реализации $v(T(x) \omega)$ потенциальны (соленоидальны) на $\mathbb{R}^{d}$. Пусть $V_{\text {pot }}^{2}$ $\left(V_{\text {pot }}^{\infty}\right)$ - это множество всех потенциальных векторов из $L^{2}(\Omega)\left(L^{\infty}(\Omega)\right)$ с нулевым средним, $\langle v\rangle=\int_{\Omega} v d \mu=0$. Известно, что $V_{\text {pot }}^{\infty}$ плотно в $V_{\text {pot }}^{2}$.

Поставим задачу: для $\xi \in \mathbb{R}^{d}$ найти вектор $v \in V_{\text {pot }}^{2}$ такой, что

$$
\int_{\Omega} A(\omega)(\xi+v(\omega)) \cdot w(\omega) d \mu=0 \quad \forall w \in V_{\mathrm{pot}}^{\infty},
$$


или в краткой записи

$$
v \in V_{\mathrm{pot}}^{2}, \quad\langle A(\xi+v) \cdot w\rangle=0 \quad \forall w \in V_{\mathrm{pot}}^{\infty} .
$$

В случае $A \in L^{\infty}(\Omega)$ существование и единственность решения этой задачи непосредственно следует из леммы Лакса-Мильграма, а усредненная матрица $A_{0}$ определяется равенством

$$
A_{0} \xi=\langle A(\xi+v)\rangle
$$

причем эту матрицу можно считать симметрической.

Установим некоторые оценки для решения задачи (6.2). Из (6.2), (6.3) имеем

$$
\begin{aligned}
A_{0} \xi \cdot \xi & =\langle A(\xi+v) \cdot \xi\rangle=\langle A(\xi+v) \cdot(\xi+v)\rangle \\
& =\langle a(\xi+v) \cdot(\xi+v)\rangle \geqslant \nu\langle(\xi+v) \cdot(\xi+v)\rangle \geqslant \nu \xi^{2} .
\end{aligned}
$$

Кроме того,

$$
\begin{gathered}
\langle A(\xi+v) \cdot v\rangle=0, \quad\langle a v \cdot v\rangle=-\langle A \xi \cdot v\rangle, \\
\|v\|_{L^{2}(\Omega)} \leqslant \nu^{-1}\|A\|_{L^{2}(\Omega)}|\xi| .
\end{gathered}
$$

Пусть теперь $B \in L^{2}(\Omega), B_{n} \rightarrow B$ в $L^{2}(\Omega), B_{n} \in L^{\infty}(\Omega)$. Тогда последовательность решений $v_{n}$ ограничена в $L^{2}(\Omega)$ и (с точностью до выделения подпоследовательности) $v_{n} \rightarrow v$ в $L^{2}(\Omega)$. Переходом к пределу в тождестве

$$
\left\langle A_{n}\left(\xi+v_{n}\right) \cdot w\right\rangle=0 \quad \forall w \in V_{\mathrm{pot}}^{\infty}
$$

получаем "аппроксимационное" решение задачи (6.2). При этом усредненная матрица определяется как соответствующий предел усредненных матриц:

$$
A_{0} \xi=\lim _{n \rightarrow \infty}\left\langle A_{n}\left(\xi+v_{n}\right)\right\rangle=\langle A(\xi+v)\rangle .
$$

Посмотрим, что представляет собой задача (6.2) на реализациях. Здесь важно следующее утверждение, см. [3; гл. VI].

Лемма 6.1. Вектор $h \in L^{1}(\Omega)^{d}$ соленоидален тогда и толъко тогда, когда он ортогонален пространству $V_{\mathrm{pot}}^{\infty}$, m.e.

$$
\int_{\Omega} h \cdot v d \mu=0 \quad \forall v \in V_{\mathrm{pot}}^{\infty}
$$

Теперь из (6.2) видим, что вектор $h=A(\xi+v)$ соленоидален, и поэтому для $\mu$-почти всех $\omega$ выполнено

$$
\operatorname{div}_{x}(A(T(x) \omega)(\xi+v(T(x) \omega)))=0 \quad \text { в } \quad \mathbb{R}^{d} .
$$

Вектор $v(x)=v(T(x) \omega)$ по определению имеет потенциал $v(x)=\nabla u(x), u \in$ $H_{\mathrm{loc}}^{1}\left(\mathbb{R}^{d}\right)$, но $u(x, \omega)$ вообще не будет стохастически однородной функцией, т.е. не допускает представления в виде $u(T(x) \omega)$, где $u \in L^{1}(\Omega)$. Указанное представление имеет место в периодическом случае, что приводит к известной задаче на ячейке периодичности:

$$
u \in H_{\text {per }}^{1}(\square), \quad \operatorname{div}(A(\xi+\nabla u))=0 .
$$


Задачу (6.2) на вероятностном пространстве по аналогии также называют "задачей на ячейке". В силу леммы 6.1 она может быть представлена в виде

$$
v \in V_{\text {pot }}^{2}, \quad \operatorname{div}(A(\xi+v))=0,
$$

где запись $\operatorname{div} g=0, g \in L^{1}(\Omega)^{d}$, означает, что вектор $g$ соленоидален.

Отметим следующее утверждение о единственности.

Tеорема 6.2. Пусть $B \in L^{2}(\Omega)$ и для почти всех реализаиий $B(x)=$ $B(T(x) \omega)$ выполнено условие (5.1). Тогда решение задачи (6.2) единственно.

Эта теорема, по существу, доказана в [19]. В этой работе предполагалось, что $B \in L^{2}(\Omega)$ и для некоторого вектора $b \in L^{2}(\Omega)$ выполнено

$$
\operatorname{div} B(T(x) \omega)=b(T(x) \omega) \quad \text { для } \mu \text {-почти всех } \omega .
$$

Однако в самом доказательстве использовалось более слабое “локальное" условие (5.1).

6.3. Наш основной результат по усреднению составляет

Теорема 6.3. Пусть $B \in L^{p}(\Omega)$ для некоторого $p>d$, а началъное значение $f$ удовлетворяет условиям (3.11). Тогда для $\mu$-почти всех $\omega$ выполнено соотношение $(6.1)$, в котором $u(x, t, \cdot)$ - аппроксимачионное решение задачи Коши, а также более сильное соотношение

$$
\lim _{\varepsilon \rightarrow 0} \int_{\mathbb{R}^{d}}\left(x^{2}+1\right)^{\lambda}\left|\varepsilon^{-d} u\left(\varepsilon^{-1} x, \varepsilon^{-2} t, \omega\right)-K_{0}(x, t)\right| d x=0 \quad \forall t>0, \quad \lambda>0 .
$$

Матрица эфбективной диффузии зависит от способа аппроксимации.

Несколько слов об аппроксимации, которая предполагается в теореме 6.3. Рассматривается последовательность $B_{n} \in L^{\infty}(\Omega), B_{n} \rightarrow B$ в $L^{p}(\Omega)$. Для почти всех реализаций $B_{n}(x)=B(T(x) \omega), B(x)=B(T(x) \omega)$ по эргодической теореме имеем сходимость $B_{n} \rightarrow B$ в $L_{\text {loc }}^{p}\left(\mathbb{R}^{d}\right)$. Нужно еще убедиться, что условие (1.4) выполняется равномерно по $n$. С этой целью воспользуемся известным результатом из эргодической теории [18; гл. VIII].

МАКСИМАЛЬНАЯ ЭРГОДИЧЕСКАЯ ТЕОРЕМА. Если $h \in L^{1}(\Omega) u$

$$
l(\omega)=\sup _{r \geqslant 1} \frac{1}{r^{d}} \int_{|x| \leqslant r}|h(T(x) \omega)| d x,
$$

то выполнено неравенство

$$
\mu\{\omega \in \Omega, l(\omega) \geqslant \lambda\} \leqslant \frac{c}{\lambda} \int_{\Omega}|h| d \mu, \quad c=c(d) .
$$

Отсюда получаем, что если $h_{n} \rightarrow h$ в $L^{1}(\Omega)$, то (с точностью до выбора подпоследовательности) $l_{n}(\omega) \rightarrow l(\omega)$ для $\mu$-почти всех $\omega$. Остается применить это свойство к последовательности $h_{n}=\left|B_{n}\right|^{p}$.

В основе доказательства теоремы 6.3 лежит оценка (1.5) для функции $\varepsilon^{-d} u\left(\varepsilon^{-1} x, \varepsilon^{-2} t, \omega\right)$ и следующая теорема об усреднении.

Пусть $w_{\varepsilon}(x, t, \omega)$ - решение задачи Коши

$$
\frac{\partial w_{\varepsilon}}{\partial t}=\operatorname{div} A\left(T\left(\varepsilon^{-1} x\right) \omega\right) \nabla w_{\varepsilon},\left.\quad w_{\varepsilon}\right|_{t=0}=\varphi(x) \in L^{2}\left(\mathbb{R}^{d}\right) .
$$


TeOpema 6.4. Пусть $B \in L^{d}(\Omega)$ npu $d \geqslant 3 u B \in L^{2+\sigma}(\Omega), \sigma>0, n p u d=2$. Тогда для н-почти всех $\omega$ для аппроксимационного решения задачи Коши (6.5) выполнено соотношение

$$
\lim _{\varepsilon \rightarrow 0} \int_{\mathbb{R}^{d}}\left|w_{\varepsilon}(x, t, \omega)-w_{0}(x, t)\right|^{2} d x=0 \quad \forall t \geqslant 0,
$$

в котором $w_{0}(x, t)$ - решение усредненной задачи Коши

$$
\frac{\partial w_{0}}{\partial t}=\operatorname{div} A_{0} \nabla w_{0},\left.\quad w_{0}\right|_{t=0}=\varphi .
$$

Усредненная матрица $A_{0}$ зависит от способа аппроксимации.

ДОКАЗАТЕЛЬСТВо тЕОРЕМЫ 6.3. Плотность

$$
u^{\varepsilon}(x, t)=u^{\varepsilon}(x, t, \omega)=\varepsilon^{-d} u\left(\varepsilon^{-1} x, \varepsilon^{-2} t, \omega\right)
$$

служит решением задачи Коши с матрицей $A\left(\varepsilon^{-1} x\right)$ и дельтаобразным начальным значением $\varepsilon^{-d} f\left(\varepsilon^{-1} x\right)$. Непосредственно теорема усреднения здесь не применима, поскольку семейство начальных значений $\varepsilon^{-d} f\left(\varepsilon^{-1} x\right)$ не ограничено в $L^{2}\left(\mathbb{R}^{d}\right)$. Однако благодаря оценке $(1.5)$ значение $u^{\varepsilon}\left(x, t_{0}\right)$, где $t_{0}>0$, принадлежит пространству $L^{2}\left(\mathbb{R}^{d}\right)$ и, более того, семейство $u^{\varepsilon}\left(x, t_{0}\right)$ компактно в $L^{2}\left(\mathbb{R}^{d}\right)$ для почти всех $t_{0}>0$ в силу леммы 3.2. Следовательно, теорема усреднения применима при $t \geqslant t_{0}>0$ и можно считать, что имеет место сильная сходимость

$$
u^{\varepsilon}(x, t) \rightarrow u^{0}(x, t) \quad \text { в } \quad L^{2}\left(\mathbb{R}^{d}\right), \quad t>0,
$$

причем $u^{0}$ есть решение усредненного уравнения $\partial u^{0} / \partial t=\operatorname{div} A_{0} \nabla u^{0}$. Остается проверить, что $u^{0}(x, 0)=\delta(x)$, т.е.

$$
\lim _{t \rightarrow 0} \int_{\mathbb{R}^{d}} u^{0}(x, t) \varphi(x) d x=\varphi(0) \quad \forall \varphi \in C_{0}^{\infty}\left(\mathbb{R}^{d}\right) .
$$

С этой целью воспользуемся оценкой (1.5) и свойством (3.12) равномерного дельтаобразного поведения. Так как

$$
\int_{\mathbb{R}^{d}} u^{\varepsilon}(x, t) d x \equiv 1
$$

то из (3.12) и (1.5) имеем

$$
\begin{aligned}
\int_{\mathbb{R}^{d}} u^{0}(x, t) d x & =\lim _{\varepsilon \rightarrow 0} \int_{\mathbb{R}^{d}} u^{\varepsilon}(x, t) d x \equiv 1, \\
\int_{|x| \geqslant r} u^{0}(x, t) d x & =\lim _{\varepsilon \rightarrow 0} \int_{|x|>r} u^{\varepsilon}(x, t) d x \leqslant c_{r}(t), \quad \lim _{t \rightarrow 0} c_{r}(t)=0 \quad \forall r>0 .
\end{aligned}
$$

Отсюда следует равенство $\lim _{t \rightarrow 0} u^{0}(x, t)=\delta(x)$, т.е. $u^{0}(x, t)=K_{0}(x, t)-$ фундаментальное решение для усредненной задачи Коши. Наконец, само соотношение (6.4) легко получается из (6.6) и оценки (1.5). Теорема 6.3 доказана. 


\section{§ 7. Доказательство теоремы об усреднении}

Установим усреднение для эллиптического уравнения

$$
-\operatorname{div} A^{\varepsilon}(x) \nabla u^{\varepsilon}+u^{\varepsilon}=f \in L^{2}\left(\mathbb{R}^{d}\right),
$$

где $A^{\varepsilon}(x)=A(T(y) \omega), y=\varepsilon^{-1} x$, а именно докажем соотношение

$$
u^{\varepsilon} \rightarrow u^{0} \quad \text { в } L^{2}\left(\mathbb{R}^{d}\right),
$$

в котором $u^{\varepsilon}-$ аппроксимационное решение указанного уравнения, а $u^{0}$ - peшение усредненного уравнения

$$
-\operatorname{div} A_{0} \nabla u^{0}+u^{0}=f .
$$

После этого сама теорема 6.4 будет следовать из теоремы Троттера-Като.

Энергетическая оценка

$$
\left\|u^{\varepsilon}\right\|_{H^{1}\left(\mathbb{R}^{d}\right)} \leqslant \nu^{-1}\|f\|_{L^{2}\left(\mathbb{R}^{d}\right)}
$$

показывает, что соотношение (7.2) достаточно доказать для любого $f \in C_{0}^{\infty}\left(\mathbb{R}^{d}\right)$. В этом случае по принципу максимума

$$
\left\|u^{\varepsilon}\right\|_{L^{\infty}\left(\mathbb{R}^{d}\right)} \leqslant\|f\|_{L^{\infty}\left(\mathbb{R}^{d}\right)} .
$$

Пусть для определенности $d \geqslant 3$. Из оценок (7.3) и (4.3) следует, что последовательность $u^{\varepsilon}$ компактна в $L^{2}\left(\mathbb{R}^{d}\right)$. Таким образом, без потери общности можно считать, что имеет место соотношение (7.2), а также справедливы соотношения

$$
u^{\varepsilon} \rightarrow u^{0} \quad \text { в } H^{1}\left(\mathbb{R}^{d}\right), \quad u^{\varepsilon} \rightarrow u^{0} \quad \text { в } \quad L_{\mathrm{loc}}^{s}\left(\mathbb{R}^{d}\right) \quad \forall s \geqslant 1,
$$

см. (7.4). Семейство $A^{\varepsilon}$ ограничено в $L_{\text {loc }}^{d}\left(\mathbb{R}^{d}\right)$, поскольку $A \in L^{d}(\Omega)$. Поэтому семейство $p^{\varepsilon}(x)=A^{\varepsilon}(x) \nabla u^{\varepsilon}(x)$ ограничено в $L_{\text {loc }}^{2 d /(d+2)}\left(\mathbb{R}^{d}\right)$ и без потери общности считаем, что

$$
p^{\varepsilon} \rightarrow p^{0} \quad \text { в } \quad L_{\mathrm{loc}}^{2 d /(d+2)}\left(\mathbb{R}^{d}\right) .
$$

Интегральное тождество для исходной задачи (7.1) запишется как

$$
\int_{\mathbb{R}^{d}}\left(p^{\varepsilon} \cdot \nabla \varphi+u^{\varepsilon} \varphi\right) d x=\int_{\mathbb{R}^{d}} f \varphi d x, \quad \varphi \in C_{0}^{\infty}\left(\mathbb{R}^{d}\right) .
$$

Будем использовать задачу на ячейке вида (6.2), отвечающую транспонированной к $A$ матрице. Запишем эту задачу в виде

$$
v \in V_{\mathrm{pot}}^{2}, \quad \operatorname{div}(\xi+v) A=0
$$

и положим

$$
v^{\varepsilon}(x)=v(T(y) \omega), \quad h=(\xi+v) A, \quad h^{\varepsilon}(x)=h(T(y) \omega), \quad y=\varepsilon^{-1} x .
$$

Тогда $\xi A_{0}=\langle h\rangle$,

$$
\operatorname{div} h^{\varepsilon}=0, \quad h^{\varepsilon} \rightarrow\langle h\rangle=\xi A_{0} \quad \text { в } \quad L_{\mathrm{loc}}^{2 d /(d+2)}\left(\mathbb{R}^{d}\right) .
$$


Вектор $v^{\varepsilon}$ потенциален и $v^{\varepsilon} \rightarrow\langle v\rangle=0$ в $L_{\mathrm{loc}}^{2}\left(\mathbb{R}^{d}\right)$ по эргодической теореме, следовательно, справедливо представление

$$
v^{\varepsilon}=\nabla w^{\varepsilon}, \quad w^{\varepsilon} \rightarrow w \quad \text { в } \quad H_{\mathrm{loc}}^{1}\left(\mathbb{R}^{d}\right) .
$$

Исходим из очевидного поточечного равенства

$$
\Phi^{\varepsilon}(x) \equiv p^{\varepsilon}(x) \cdot \nabla w^{\varepsilon}(x)=\nabla u^{\varepsilon}(x) \cdot v^{\varepsilon}(x) A^{\varepsilon}(x)=\nabla u^{\varepsilon}(x) \cdot h^{\varepsilon}(x)-p^{\varepsilon}(x) \cdot \xi .
$$

При наших условиях на матрицу $A$ функция $\Phi^{\varepsilon}(x)$, вообще говоря, не принадлежит $L_{\mathrm{loc}}^{1}\left(\mathbb{R}^{d}\right)$. Поэтому преобразуем величину

$$
J_{\varepsilon}=\int_{\mathbb{R}^{d}} \Phi^{\varepsilon}(x) \varphi(x) d x, \quad \varphi \in C_{0}^{\infty}\left(\mathbb{R}^{d}\right),
$$

предполагая пока, что $A \in L^{\infty}(\Omega)$. Из (7.5) имеем

$$
J_{\varepsilon} \equiv \int_{\mathbb{R}^{d}} p^{\varepsilon} \cdot \nabla w^{\varepsilon} \varphi d x=\int_{\mathbb{R}^{d}}\left(f-u^{\varepsilon}\right) w^{\varepsilon} \varphi d x-\int_{\mathbb{R}^{d}} p^{\varepsilon} \cdot w^{\varepsilon} \nabla \varphi d x .
$$

Кроме того,

$$
J_{\varepsilon}=\int_{\mathbb{R}^{d}} \nabla u^{\varepsilon} \cdot h^{\varepsilon} \varphi d x-\int_{\mathbb{R}^{d}} p^{\varepsilon}(x) \cdot \xi \varphi d x=-\int_{\mathbb{R}^{d}} u^{\varepsilon} \cdot h^{\varepsilon} \nabla \varphi d x-\int_{\mathbb{R}^{d}} p^{\varepsilon}(x) \cdot \xi \varphi d x
$$

в силу соленоидальности вектора $h^{\varepsilon}$.

В результате получим равенство

$$
-\int_{\mathbb{R}^{d}} p^{\varepsilon} \cdot w^{\varepsilon} \nabla \varphi d x+\int_{\mathbb{R}^{d}}\left(f-u^{\varepsilon}\right) w^{\varepsilon} \varphi d x+\int_{\mathbb{R}^{d}} p^{\varepsilon} \cdot \xi \varphi d x+\int_{\mathbb{R}^{d}} u^{\varepsilon} h^{\varepsilon} \cdot \nabla \varphi d x=0 .
$$

Теперь нужно проверить, что это равенство верно для аппроксимационных решений, а затем перейти в нем к пределу при $\varepsilon \rightarrow 0$. Оба предельных перехода основаны на одинаковых соображениях, причем некоторую трудность составляет только первое слагаемое - в остальных предельный переход основан на простейших свойствах сильной и слабой сходимости. Начнем с предельного перехода при фиксированном $\varepsilon>0$. В равенстве $(7.7)$ вместо $u^{\varepsilon}, p^{\varepsilon}, \ldots$ надо взять $u_{n}^{\varepsilon}, p_{n}^{\varepsilon}, \ldots$. При переходе к пределу в задаче на ячейке имеем слабую сходимость

$$
v_{n} \rightarrow v \quad \text { в } L^{2}(\Omega), \quad h_{n} \rightarrow h \quad \text { в } L^{2 d /(d+2)}(\Omega),
$$

которая влечет слабую сходимость реализаций

$$
\begin{array}{lll}
v_{n}\left(T\left(\varepsilon^{-1} x\right) \omega\right) \rightarrow v\left(T\left(\varepsilon^{-1} x\right) \omega\right) & \text { в } \quad L_{\mathrm{loc}}^{2}\left(\mathbb{R}^{d}\right), \\
h_{n}\left(T\left(\varepsilon^{-1} x\right) \omega\right) \rightarrow h\left(T\left(\varepsilon^{-1} x\right) \omega\right) & \text { в } \quad L_{\mathrm{loc}}^{2 d /(d+2)}\left(\mathbb{R}^{d}\right)
\end{array}
$$

при фиксированном $\varepsilon$ (для $\mu$-почти всех $\omega)$.

В результате при $n \rightarrow \infty$ имеем:

1) $p_{n}^{\varepsilon} \rightarrow p^{\varepsilon}, h_{n}^{\varepsilon} \rightarrow h^{\varepsilon}$ в $L_{\text {loc }}^{2 d /(d+2)}\left(\mathbb{R}^{d}\right)$,

2) $u_{n}^{\varepsilon} \rightarrow u^{\varepsilon}$ в $L_{\mathrm{loc}}^{s}\left(\mathbb{R}^{d}\right)(\forall s \geqslant 1), w_{n}^{\varepsilon} \rightarrow w^{\varepsilon}$ в $H_{\mathrm{loc}}^{1}\left(\mathbb{R}^{d}\right)$. 
Отсюда видно, что во всех слагаемых в (7.7), кроме первого, предельный переход совсем прост.

Напомним, что последовательность измеримых функций $\beta_{n}(x)$ на $Q(Q$-шар в $\mathbb{R}^{d}$ ) равностепенно интегрируема, если для любого $\delta>0$ найдется $\tau=\tau(\delta)$ такое, что для любого измеримого множества $K \subset Q$

$$
\int_{K}\left|\beta_{n}(x)\right| d x \leqslant \delta
$$

как только $|K| \leqslant \tau$, где $|K|$ - мера Лебега множества $K$.

Известно, что последовательность $\beta_{n}$ слабо компактна в $L^{1}(Q)$ тогда и только тогда, когда она равностепенно интегрируема.

Вернемся к первому слагаемому в (7.7). Легко видеть, что

$$
\lim _{n \rightarrow \infty} \int_{\mathbb{R}^{d}} p_{n}^{\varepsilon} \cdot w_{n}^{\varepsilon} \nabla \varphi d x=\int_{\mathbb{R}^{d}} p^{\varepsilon} \cdot w^{\varepsilon} \nabla \varphi d x+\lim _{n \rightarrow \infty} \int_{\mathbb{R}^{d}} p_{n}^{\varepsilon} \cdot\left(w_{n}^{\varepsilon}-w^{\varepsilon}\right) \nabla \varphi d x .
$$

По неравенству Гёльдера

$$
\begin{aligned}
& \int_{K}\left|p_{n}^{\varepsilon}\left(w_{n}^{\varepsilon}-w\right)\right| d x \leqslant \int_{K}\left|A_{n}^{\varepsilon}\right|\left|\nabla u_{n}^{\varepsilon}\right|\left|w_{n}^{\varepsilon}-w\right| d x \\
& \quad \leqslant\left(\int_{K}\left|A_{n}^{\varepsilon}\right|^{d} d x\right)^{1 / d}\left(\int_{Q}\left|\nabla u_{n}^{\varepsilon}\right|^{2} d x\right)^{1 / 2}\left(\int_{Q}\left|w_{n}^{\varepsilon}-w\right|^{2 d /(d-2)} d x\right)^{(d-2) / d} \\
& \quad \leqslant c\left(\int_{K}\left|A_{n}^{\varepsilon}\right|^{d} d x\right)^{1 / d} .
\end{aligned}
$$

Отсюда следует, что последовательность $\left|p_{n}^{\varepsilon}\left(w_{n}^{\varepsilon}-w\right)\right|$ равностепенно интегрируема, поскольку таковой является $\left|A_{n}^{\varepsilon}\right|^{d}$ в силу сходимости $A_{n}^{\varepsilon} \rightarrow A^{\varepsilon}$ в $L^{d}(Q)$.

Рассмотрим второе слагаемое в (7.8). Положив для ясности $a_{n}=\left|p_{n}^{\varepsilon}\right|, b_{n}=$ $\left|w_{n}^{\varepsilon}-w^{\varepsilon}\right|$, введем срезку

$$
a_{n}^{N}= \begin{cases}a_{n}, & \text { если } a_{n}(x) \leqslant N, \\ N, & \text { если } a_{n}(x)>N,\end{cases}
$$

и пусть $K_{n}^{N}=\left\{x \in Q, a_{n}(x)>N\right\}$. Справедлива оценка $\left|K_{n}^{N}\right| \leqslant c N^{-1}$, вытекающая из ограниченности $a_{n}$ в $L^{1}(Q)$. Имеем

$$
\int_{\Omega} a_{n} b_{n} d x=\int_{\Omega \backslash K_{n}^{N}} a_{n}^{N} b_{n} d x+\int_{K_{n}^{N}} a_{n} b_{n} d x .
$$

При фиксированном $N$ первый интеграл справа сходится к нулю, поскольку $b_{n} \rightarrow 0$ в $L^{2}(Q)$. Второй интеграл (в силу равностепенной интегрируемости последовательности $\left.a_{n} b_{n}\right)$ можно сделать сколь угодно малым за счет выбора $N$ равномерно по $n$. Отсюда следует соотношение $a_{n} b_{n} \rightarrow 0$ в $L^{1}(Q)$. Таким образом, второе слагаемое в (7.8) равно нулю и равенство (7.7) справедливо для аппроксимационных решений.

Теперь перейдем к пределу в (7.7) при $\varepsilon \rightarrow 0$. Очевидно, что

$$
\lim _{\varepsilon \rightarrow 0} \int_{\mathbb{R}^{d}}\left(f-u^{\varepsilon}\right) w^{\varepsilon} \varphi=0
$$


так как семейство $\left(f-u^{\varepsilon}\right)$ ограничено в $L^{2}\left(\mathbb{R}^{d}\right)$, а $w^{\varepsilon} \rightarrow 0$ в $L_{\mathrm{loc}}^{2}\left(\mathbb{R}^{d}\right)$, см. (7.6). Кроме того,

$$
\begin{gathered}
\lim _{\varepsilon \rightarrow 0} \int_{\mathbb{R}^{d}} p^{\varepsilon} \cdot \xi \varphi d x=\int_{\mathbb{R}^{d}} p^{0} \cdot \xi \varphi d x \\
\lim _{\varepsilon \rightarrow 0} \int_{\mathbb{R}^{d}} u^{\varepsilon} \cdot h^{\varepsilon} \nabla \varphi d x=\int_{\mathbb{R}^{d}} u^{0}\langle h\rangle \nabla \varphi d x=\int_{\mathbb{R}^{d}} u^{0} \nabla \varphi \cdot \xi A_{0} d x
\end{gathered}
$$

поскольку $u^{\varepsilon} \rightarrow u$ в $L_{\text {loc }}^{2 d /(d-2)}\left(\mathbb{R}^{d}\right)$, а $h^{\varepsilon} \rightarrow\langle h\rangle=\xi A_{0}$ в $L_{\text {loc }}^{2 d /(d+2)}\left(\mathbb{R}^{d}\right)$.

Остается рассмотреть первое слагаемое в (7.7). По неравенству Гёльдера

$$
\begin{aligned}
\int_{K}\left|p^{\varepsilon} w^{\varepsilon}\right| d x & \leqslant \int_{K}\left|A^{\varepsilon}\right|\left|\nabla u^{\varepsilon}\right|\left|w^{\varepsilon}\right| d x \\
& \leqslant\left(\int_{K}\left|A^{\varepsilon}\right|^{d} d x\right)^{1 / d}\left(\int_{Q}\left|\nabla u^{\varepsilon}\right|^{2} d x\right)^{1 / 2}\left(\int_{Q}\left|w^{\varepsilon}\right|^{2 d /(d-2)} d x\right)^{(d-2) / d} \\
& \leqslant c\left(\int_{K}\left|A^{\varepsilon}\right|^{d} d x\right)^{1 / d}
\end{aligned}
$$

см. (7.6). Отсюда видно, что последовательность $p^{\varepsilon} w^{\varepsilon}$ равностепенно интегрируема, поскольку таковой является $\left|A^{\varepsilon}\right|^{d}$. Действительно, по эргодической теореме имеем слабую сходимость $\left|A^{\varepsilon}\right|^{d} \rightarrow\left\langle|A|^{d}\right\rangle$ в $L^{1}(Q)$, которая влечет равностепенную интегрируемость. Тем же рассуждением со срезкой, что и при переходе к пределу при $n \rightarrow \infty$, убеждаемся, что $\left|p_{\varepsilon}\right|\left|w_{\varepsilon}\right| \rightarrow 0$ в $L^{1}(Q)$ при $\varepsilon \rightarrow 0$. В результате

$$
\lim _{\varepsilon \rightarrow 0} \int_{\mathbb{R}^{d}} p^{\varepsilon} \cdot w^{\varepsilon} \nabla \varphi d x=0,
$$

и мы приходим (см. (7.9)-(7.11)) к соотношению

$$
\int_{\mathbb{R}^{d}} p^{\varepsilon} \cdot \xi \varphi d x+\int_{\mathbb{R}^{d}} u^{0} \nabla \varphi \cdot \xi A_{0} d x=0, \quad \varphi \in C_{0}^{\infty}\left(\mathbb{R}^{d}\right),
$$

из которого следует важное равенство

$$
p^{0}=A_{0} \nabla u^{0} .
$$

С другой стороны, из (7.5) получаем тождество

$$
\int_{\mathbb{R}^{d}}\left(p^{0} \cdot \nabla \varphi+u^{0} \varphi\right) d x=\int_{\mathbb{R}^{d}} f \varphi d x, \quad \varphi \in C_{0}^{\infty}\left(\mathbb{R}^{d}\right),
$$

так что $u^{0}$ - решение усредненного уравнения. Тем самым усреднение для эллиптического уравнения доказано.

\section{Список литературы}

[1] D. G. Aronson, "Bounds for the fundamental solutions of a parabolic equation", Bull. Amer. Math. Soc., 73 (1967), 890-896.

[2] D. G. Aronson, "Non-negative solutions of linear parabolic equations", Ann. Scuola Norm. Sup. Pisa. Cl. Sci. (4), 22 (1968), 607-694; Addendum, 25 (1971), 221-228. 
[3] В. В. Жиков, С. М. Козлов, О.А. Олейник, Усреднение дифференциальных операторов, Наука, М., 1993.

[4] В. В. Жиков, "Замечания о единственности решения задачи Дирихле для эллиптического уравнения второго порядка с младшими членами", Функи. анализ и его прил., 38:3 (2004), 15-28.

[5] Ф. О. Порпер, С. Д. Эйдельман, “Двусторонние оценки для фундаментальных решений параболических уравнений второго порядка и некоторые их приложения", УМН, 39:3 (1984), 107-156.

[6] А.К. Гущин, "О равномерной стабилизации решений второй смешанной задачи для параболического уравнения", Матем. сб., 119(161):4(12) (1982), 451-508.

[7] В. В. Жиков, "Асимптотические задачи, связанные с уравнением теплопроводности в перфорированных областях", Матем. сб., 181:10 (1990), 1283-1305.

[8] A. Grigor'yan, "Gaussian upper bounds for the heat kernel on arbitrary manifolds", J. Differential Geom., 45:1 (1997), 33-52.

[9] E. B. Davies, Heat kernels and spectral theory, Cambridge Univ. Press, Cambridge, 1989.

[10] K. Oelschläger, "Homogenization of a diffusion process in a divergence free random field", Ann. Probab., 16 (1988), 1084-1126.

[11] A. Fannjiang, G. Papanicolaou, "Diffusion in turbulence", Probab. Theory and Related Fields, 105 (1996), 279-334.

[12] A. Fannjiang, T. Komorowski, "An invariance principle for diffusion in turbulence", Ann. Probab., 27 (1999), 751-781.

[13] A. Fannjiang, T. Komorowski, "Turbulent diffusion in Markovian flows", Ann. Appl. Probab., 9 (1999), 591-610.

[14] A. Fannjiang, T. Komorowski, "A martingale approach to homogenization of unbounded random flows", Ann. Probab., 25 (1997), 1872-1894.

[15] О.А. Ладыженская, В.А. Солонников, Н. Н. Уральцева, Линейнъе и квазилинейнъе уравнения параболического типа, Наука, М., 1967.

[16] Б. М. Левитан, В. В. ЖКиков, Почти периодические функции и дифференциалънъе уравнения, Изд-во МГУ, М., 1978.

[17] Т. Като, Теория возмущений линейных операторов, Мир, М., 1972.

[18] Н. Данфорд, Дж. Шварц, Линейные операторы. Общая теория, Мир, М., 1962.

[19] В. В. Жиков, “Диффузия в несжимаемом случайном потоке”, Функи. анализ и его прил., 31:3 (1997), 10-22.

В. В. Жиков (V. V. Zhikov)

Владимирский государственный

педагогический университет

E-mail: zhikov@vgpu.vladimir.ru
Поступила в редакцию

07.12 .2005 